\title{
Nonlinear stability of cosmological solutions in massive gravity
}

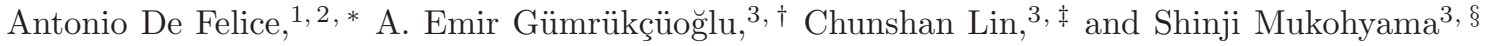 \\ ${ }^{1}$ ThEP's CRL, NEP, The Institute for Fundamental Study, \\ Naresuan University, Phitsanulok 65000, Thailand \\ ${ }^{2}$ Thailand Center of Excellence in Physics, Ministry of Education, Bangkok 10400, Thailand \\ ${ }^{3}$ Kavli Institute for the Physics and Mathematics of the Universe (WPI), \\ Todai Institutes for Advanced Study, University of Tokyo, \\ 5-1-5 Kashiwanoha, Kashiwa, Chiba 277-8583, Japan
}

(Dated: September 9, 2018)

\begin{abstract}
We investigate nonlinear stability of two classes of cosmological solutions in massive gravity: isotropic Friedmann-Lemaître-Robertson-Walker (FLRW) solutions and anisotropic FLRW solutions. For this purpose we construct the linear cosmological perturbation theory around axisymmetric Bianchi type-I backgrounds. We then expand the background around the two classes of solutions, which are fixed points of the background evolution equation, and analyze linear perturbations on top of it. This provides a consistent truncation of nonlinear perturbations around these fixed point solutions and allows us to analyze nonlinear stability in a simple way. In particular, it is shown that isotropic FLRW solutions exhibit nonlinear ghost instability. On the other hand, anisotropic FLRW solutions are shown to be ghost-free for a range of parameters and initial conditions.
\end{abstract}

\section{INTRODUCTION}

Our current understanding of gravitation is based on general relativity (GR), whose predictions are consistent with all available experimental and observational data. To this end, in many cases the so called parameterized post-Newtonian (PPN) formalism has been adopted to quantify possible deviation from GR and to compare with experiments and observations. There are indeed many theoretical models that fall into the scope of the PPN formalism. While the PPN formalism has been useful, however, it does not include finite range modification of GR. From phenomenological viewpoints, in analogy with the finite range nature of the weak interaction in the standard model of particle physics, one might argue that the possibility of finite range modification of GR should be taken into account when experimental and observational data are used to constrain deviation from GR. Additionally, such an extension is also expected to alter behavior of gravity at scales larger than the Compton wavelength of the graviton; a graviton mass as small as the current expansion rate of the universe may provide the source for the accelerated expansion alternative to dark energy.

From the theoretical point of view, construction of a finite-range gravity theory has been a long-standing problem. The massive extension of GR at the linearized level was first considered by Fierz and Pauli [1] in 1939. It was then pointed out in 1970 that the linear theory suffers from a discontinuity [2, 3] in the massless limit, now known as the vDVZ discontinuity. The discontinuity can be alleviated by allowing for nonlinear terms à la Vainshtein [4] but, as pointed out in 1972 by Boulware and Deser [5], generic nonlinear massive theories contain an unwanted sixth degree, in addition to the five polarizations of massive spin-2 field, which leads to ghost instability. Since then, it took a long time until a nonlinear extension of the Fierz-Pauli theory without the Boulware-Deser ghost, dubbed the dRGT theory, was found recently [6, 7], where the unwanted extra degree is successfully eliminated by construction [8]. However, the absence of the sixth mode does not necessarily imply that the theory is healthy. For instance, in some backgrounds, one (or more) of the five polarizations of massive graviton may be superluminal [9 11], or may become a ghost 12.

The analysis of the degrees of freedom in the dRGT theory, which originally uses the Minkowski fiducial metric, are done by considering the Minkowski background (see e.g. (7]), which resides in the "normal branch". On the other hand, for the theory to have a phenomenological relevance, it is essential to obtain non-trivial background solutions which can describe the cosmological evolution. However, in the normal branch no cosmological solution exists [13]. If the theory is extended to allow for a general fiducial metric ${ }^{1}$, expanding universe solutions can be realized in the

\footnotetext{
*Electronic address: antoniod@nu.ac.th

${ }^{\dagger}$ Electronic address: emir.gumrukcuoglu@ipmu.jp

‡Electronic address: chunshan.lin@ipmu.jp

$\S$ Electronic address: shinji.mukohyama@ipmu.jp

1 The dRGT theory extended in this way was shown to be free of Boulware-Deser instability in Ref. 14].
} 
normal branch [15 17], but the Higuchi bound 12] cannot be respected; for instance, for de Sitter reference metric, the scalar graviton becomes a ghost at expansion rates higher than the graviton mass [16]. ${ }^{2}$

In the presence of a negative spatial curvature 21] or a general reference metric [15], there exists a second branch of solutions, dubbed the "self-accelerating branch", which stems from the factorized form of the temporal Stückelberg equation of motion (or equivalently, from the Bianchi identities). With the strict Friedmann-Lemaitre-RobertsonWalker (FLRW) symmetry, this branch is disconnected from the normal branch and as a result, the dynamics of perturbations are dramatically different; even though the massive theories should have five degrees of freedom after the removal of the Boulware-Deser degree, only two degrees (the two tensor modes in the standard $\mathrm{SO}(3)$ decomposition) propagate at the linear level [15]. The resulting linear theory is thus very similar to GR, with the exception of a nonvanishing and time-dependent mass for the two gravitational wave polarizations, which may have left a characteristic signature on observables 22]. This peculiar behavior may be considered as a side-effect of the FLRW symmetry, which leads to the disconnection of the two branches. In fact, as we show in Sec. [II] the introduction of anisotropies to the background and thus deviation from the FLRW symmetry break the factorized form of the temporal Stückelberg equation, leading to a coupling between the two branches. Around such deformed backgrounds, all five graviton polarizations are generically dynamical at linear order.

From the technical point of view, one of the goals of the present paper is to construct the theory of cosmological perturbations around an axisymmetric Bianchi type-I universe in dRGT theory. From physical viewpoints, on the other hand, the goal of the paper is to investigate nonlinear stability of two distinct classes of cosmological solutions: self-accelerating isotropic FLRW solutions [15, 21] and anisotropic FLRW solutions [23]. We will achieve this goal by allowing for small deviation of the background from the above mentioned two classes of solutions, applying the general formalism to analyze perturbations around those deformed backgrounds and then considering such linear perturbations as leading nonlinear perturbations around the undeformed backgrounds. ${ }^{3}$

The resulting quadratic kinetic action, which is non-zero for all five degrees at linear order in background deformations, corresponds to the cubic kinetic terms from the perspective of undeformed backgrounds.

This approach, previously reported in 25] for isotropic FLRW solutions, reveals that the isotropic FLRW solution always has a ghost mode at nonlinear order with a gapless dispersion relation. This conclusion is valid for all homogeneous and isotropic solutions in the self-accelerating branch.

Although the deformation of the FLRW background with small anisotropy leads to an inevitable ghost mode, there may still be healthy regions with relatively large anisotropy. This is in analogy with scalar field models of ghost condensation [26, 27]. As the second application of the formalism developed in the first half of the present paper, we thus explore this possibility, by considering the anisotropic FLRW attractor solution of Ref. [23] as the undeformed background solution. On this attractor, the expansion of the physical metric is isotropic, with a de Sitter evolution. The fiducial metric also exhibits a de Sitter expansion but is actually anisotropic from the viewpoint of comoving observers residing on the physical metric. This background thus contains anisotropy which can only be probed by perturbations, whose evolution depends on the alignment of the privileged direction. Applying the general perturbation theory to this example yields that on the attractor, there are still two degrees of freedom which do not propagate at linear order. By adding homogeneous deformations to the background, we find that the perturbations can in principle have a stable behavior at nonlinear order, depending on the parameters and initial conditions. This result suggests the existence of a healthy region between the isotropic and anisotropic FLRW solutions.

The paper is organized as follows: we start by reviewing the massive gravity action in Sec II Without choosing the solution, we first present the equations for an axisymmetric Bianchi-I background in Sec. [II] and metric perturbations in Sec IV In Sec $\mathrm{V}$ we expand the background with respect to small anisotropy and discuss the implications regarding the nonlinear stability of the isotropic FLRW solution (this is a detailed calculation of the results already presented in [25]). In Sec:VT] we consider small deviations from the anisotropic fixed point solution of [23] and obtain conditions for linear and nonlinear stability of these solutions. Finally, in SecVII, we conclude the study with a discussion of our results. The paper is supplemented with several Appendices, where technical details are presented.

\section{ACTION}

We start this section by reviewing the action for nonlinear massive gravity in generic setup.

\footnotetext{
2 An exception to this conclusion may be provided if gravity is partially massless, i.e. if there exists an additional symmetry which forces the Higuchi bound to be saturated, leading to the disappearance of the scalar mode within five propagating degrees of freedom [18]. However, there are indications that such a construction cannot be obtained from the dRGT theory [19, 20].

3 This is qualitatively similar to the instability encountered in the self-accelerating solutions at the decoupling limit, arising once the vectors are turned on [24].
} 
The graviton mass is introduced through a non-derivative term by [].5],

$$
I=I_{E H, \Lambda}[g]+I_{\text {matter }}[g, \psi]+I_{\text {mass }}\left[g^{-1} f\right],
$$

where the first term is the Einstein-Hilbert action with a cosmological constant $\Lambda$

$$
I_{R H, \Lambda}[g]=\frac{M_{P l}}{2} \int d^{4} \sqrt{-g}(R-2 \Lambda),
$$

and the matter sector consists of fields $\{\psi\}$, coupled to gravity through the physical metric $g$. The existence of a massive graviton implies breaking of the general coordinate invariance, which cannot be accomplished by the physical metric $g$ alone [5]. For this task, the space-time tensor $f_{\mu \nu}$, dubbed the "fiducial metric" is introduced and parametrized as

$$
f_{\mu \nu} \equiv \bar{f}_{A B}\left(\phi^{C}\right) \partial_{\mu} \phi^{A} \partial_{\nu} \phi^{B}
$$

where the four scalar fields $\phi^{A}(A=0,1,2,3)$ are the Stückelberg fields, which are responsible for the breaking of the four gauge degrees of freedom, while $\bar{f}_{A B}\left(\phi^{C}\right)$ is the metric in the field space.

Imposing the absence of the BD ghost in the decoupling limit, the mass part in (11) can be constructed as [6]

$$
I_{\text {mass }}\left[g^{-1} f\right]=M_{p}^{2} m_{g}^{2} \int d^{4} x \sqrt{-g}\left(\mathcal{L}_{2}+\alpha_{3} \mathcal{L}_{3}+\alpha_{4} \mathcal{L}_{4}\right)
$$

with

$$
\begin{aligned}
\mathcal{L}_{2} & =\frac{1}{2}\left([\mathcal{K}]^{2}-\left[\mathcal{K}^{2}\right]\right) \\
\mathcal{L}_{3} & =\frac{1}{6}\left([\mathcal{K}]^{3}-3[\mathcal{K}]\left[\mathcal{K}^{2}\right]+2\left[\mathcal{K}^{3}\right]\right) \\
\mathcal{L}_{4} & =\frac{1}{24}\left([\mathcal{K}]^{4}-6[\mathcal{K}]^{2}\left[\mathcal{K}^{2}\right]+3\left[\mathcal{K}^{2}\right]^{2}+8[\mathcal{K}]\left[\mathcal{K}^{3}\right]-6\left[\mathcal{K}^{4}\right]\right)
\end{aligned}
$$

where the square brackets denote the trace operation and

$$
\mathcal{K}_{\nu}^{\mu}=\delta_{\nu}^{\mu}-\left(\sqrt{g^{-1} f}\right)_{\nu}^{\mu}
$$

\section{ANISOTROPIC BACKGROUND}

In this section, we obtain the background field equations for an anisotropic extension of the FLRW physical metric. ${ }^{4}$ Since one of our goals with this extension is to obtain the stability conditions of the isotropic metric, in the isotropic limit, the whole system needs to reduce to the cosmological solutions in [15, 21]. For this reason, we consider a fiducial metric that is of the flat FLRW form,

$$
f_{\mu \nu}=-n^{2}\left(\phi^{0}\right) \partial_{\mu} \phi^{0} \partial_{\nu} \phi^{0}+\alpha^{2}\left(\phi^{0}\right)\left(\partial_{\mu} \phi^{1} \partial_{\nu} \phi^{1}+\delta_{i j} \partial_{\mu} \phi^{i} \partial_{\nu} \phi^{j}\right)
$$

where nonzero expectation value of the Stückelberg fields coincide with the space-time coordinates $\left\langle\phi^{0}\right\rangle=t,\left\langle\phi^{1}\right\rangle=$ $x,\left\langle\phi^{i}\right\rangle=y^{i}$. In the rest of the paper, Greek indices span the space-time coordinates, while lower case latin indices $i, j=2,3$ correspond to the coordinates on the $y-z$ plane, with $y^{2}=y, y^{3}=z$.

The physical metric is chosen to be the simplest anisotropic extension of FLRW, namely, the axisymmetric Bianchi type-I metric

$$
g_{\mu \nu}^{(0)} d x^{\mu} d x^{\nu}=-N^{2}(t) d t^{2}+a^{2}(t)\left(e^{4 \sigma(t)} d x^{2}+e^{-2 \sigma(t)} \delta_{i j} d y^{i} d y^{j}\right) .
$$

Varying the Stückelberg fields around the background values

$$
\phi^{A}=x^{A}+\pi^{A},
$$

\footnotetext{
${ }^{4}$ For a more general discussion of Bianchi class A cosmologies in the context of bigravity, see [28].
} 
the mass term action up to first order in $\pi^{a}$ becomes

$$
I_{\text {mass }}=I_{\text {mass }}^{(0)}+M_{P l}^{2} m_{g}^{2} \int d^{4} x N a^{3} n \pi^{0} \mathcal{E}_{\phi}+\mathcal{O}\left[\left(\pi^{a}\right)^{2}\right],
$$

giving the background equation of motion for the Stückelberg fields as

$$
\mathcal{E}_{\phi} \equiv J_{\phi}^{(x)}\left(H+2 \Sigma-H_{f} e^{-2 \sigma} X\right)+2 J_{\phi}^{(y)}\left(H-\Sigma-H_{f} e^{\sigma} X\right)=0,
$$

where

$$
H \equiv \frac{\dot{a}}{a N}, \quad H_{f} \equiv \frac{\dot{\alpha}}{\alpha n}, \quad \Sigma \equiv \frac{\dot{\sigma}}{N}, \quad X \equiv \frac{\alpha}{a},
$$

and

$$
\begin{aligned}
& J_{\phi}^{(x)} \equiv 3-2 e^{\sigma} X+\alpha_{3}\left(3-4 e^{\sigma} X+e^{2 \sigma} X^{2}\right)+\alpha_{4}\left(1-e^{\sigma} X\right)^{2} \\
& J_{\phi}^{(y)} \equiv 3-e^{-2 \sigma} X-e^{\sigma} X+\alpha_{3}\left(3-2 e^{-2 \sigma} X-2 e^{\sigma} X+e^{-\sigma} X^{2}\right)+\alpha_{4}\left(1-e^{-2 \sigma} X\right)\left(1-e^{\sigma} X\right)
\end{aligned}
$$

The expansion rate for the fiducial metric $H_{f}$ is related to the invariants of the field space metric $\bar{f}_{a b}\left(\phi^{C}\right)$ which are fixed by the theory, and is independent of the choice of the background values of $\phi^{a}$. Thus, Eq. (11) can be interpreted as an algebraic equation for $\alpha$ (or equivalently for $X$ ), but not a differential equation. We also note that, in the isotropic limit $(\sigma, \Sigma \rightarrow 0)$, we have $J_{\phi}^{(x)}=J_{\phi}^{(y)}$, and the corresponding equation in the FLRW case (c.f. Eq.(A.11) of Ref. [15]) is recovered.

Finally, we calculate the equations of motion for the metric $g_{\mu \nu}$. Since we are interested in the stability of the gravity sector only, we neglect any matter field and consider only the vacuum configuration. The independent components of the field equations for the physical metric are

$$
\begin{array}{r}
\begin{aligned}
3\left(H^{2}-\Sigma^{2}\right)-\Lambda=m_{g}^{2}\left[-\left(6+4 \alpha_{3}+\alpha_{4}\right)+\left(3+3 \alpha_{3}+\alpha_{4}\right)\left(2 e^{\sigma}+e^{-2 \sigma}\right) X\right. \\
\left.-\left(1+2 \alpha_{3}+\alpha_{4}\right)\left(e^{2 \sigma}+2 e^{-\sigma}\right) X^{2}+\left(\alpha_{3}+\alpha_{4}\right) X^{3}\right], \\
3\left(\frac{\dot{\Sigma}}{N}+3 H \Sigma\right)=m_{g}^{2}\left(e^{-2 \sigma}-e^{\sigma}\right) X\left[\left(3+3 \alpha_{3}+\alpha_{4}\right)-\left(1+2 \alpha_{3}+\alpha_{4}\right)\left(e^{\sigma}+r\right) X+\left(\alpha_{3}+\alpha_{4}\right) r e^{\sigma} X^{2}\right], \\
-6\left(\frac{\dot{H}}{N}+3 \Sigma^{2}\right)=m_{g}^{2} X\left[\left(3+3 \alpha_{3}+\alpha_{4}\right)\left(2 e^{\sigma}+e^{-2 \sigma}-3 r\right)+\left(\alpha_{3}+\alpha_{4}\right)\left[3-\left(e^{2 \sigma}+2 e^{-\sigma}\right) r\right] X^{2}\right. \\
\left.+2\left(1+2 \alpha_{3}+\alpha_{4}\right)\left[\left(2 e^{\sigma}+e^{-2 \sigma}\right) r-\left(e^{2 \sigma}+2 e^{-\sigma}\right)\right] X\right],
\end{aligned}
\end{array}
$$

where we have introduced

$$
r \equiv \frac{n}{N X}
$$

\section{PERTURBATIONS}

In this section, we calculate the action quadratic in perturbations around the metric (8). The most general set of perturbations around the axisymmetric Bianchi type-I are given by [29]

$$
g_{\mu \nu}^{(1)}=\left(\begin{array}{rrr}
-2 N^{2} \Phi & a e^{2 \sigma} N \partial_{x} \chi & a e^{-\sigma} N\left(\partial_{i} B+v_{i}\right) \\
a^{2} e^{4 \sigma} \psi & a^{2} e^{\sigma} \partial_{x}\left(\partial_{i} \beta+\lambda_{i}\right) \\
& & a^{2} e^{-2 \sigma}\left[\tau \delta_{i j}+\partial_{i} \partial_{j} E+\partial_{i} h_{j}\right)
\end{array}\right)
$$

where $\partial_{(i} h_{j)} \equiv\left(\partial_{i} h_{j}+\partial_{i} h_{j}\right) / 2$ and $\partial^{i} v_{i}=\partial^{i} \lambda_{i}=\partial^{i} h_{i}=0$. Note that, since the $y-z$ plane is Euclidean, the indices $i, j$ are raised and lowered with $\delta^{i j}$ and $\delta_{i j}$. Similarly, we decompose the perturbations of the Stückelberg fields (9) as

$$
\pi^{A}=\left(\pi^{0}, \partial_{1} \pi^{1}, \partial^{i} \pi+\pi^{i}\right)
$$


where $\partial_{i} \pi^{i}=0$. Since the vectors are defined on the $2 \mathrm{~d} y-z$ plane, the transversity condition can be used to reduce each of these vectors to a single degree of freedom

$$
v_{i}=\epsilon_{i}^{j} \partial_{j} v, \quad \lambda_{i}=\epsilon_{i}^{j} \partial_{j} \lambda, \quad h_{i}=\epsilon_{i}^{j} \partial_{j} h, \quad \pi_{i}=\epsilon_{i}^{j} \partial_{j} \pi_{\text {odd }},
$$

where $\epsilon_{i j}$ is a unit anti-symmetric tensor with $\epsilon_{23}=-\epsilon_{32}=1$ and $\epsilon_{i}^{j}=\epsilon_{i k} \delta^{k j}$.

Although the nonzero $\sigma$ in the background metric (8) breaks the isotropy, there is still a residual symmetry corresponding to rotations around the $x$ axis. Under such a rotation, the perturbations which transform as $2 \mathrm{~d}$ scalars, or even modes $(\Phi, \chi, B, \psi, \beta, \tau, E)$ and the perturbations which transform as $2 \mathrm{~d}$ vectors, or odd modes $(v, \lambda, h)$ decouple at the level of the quadratic action. This allows us to study each sector separately in the following.

\section{A. Gauge invariant variables}

Before moving on to the discussion of the action, let us first determine the transformation properties of the perturbations and build gauge invariant variables. We consider infinitesimal coordinate transformations

$$
x^{\mu} \rightarrow x^{\mu}+\xi^{\mu},
$$

with

$$
\xi^{A}=\left(\xi^{0}, \partial_{1} \xi^{1}, \partial^{i} \xi+\epsilon^{i j} \partial_{j} \xi_{\text {odd }}\right)
$$

Under this transformation, the even perturbations transform as

$$
\begin{aligned}
\Phi & \rightarrow \Phi+\frac{1}{N} \partial_{t}\left(N \xi^{0}\right), \\
\chi & \rightarrow \chi-\frac{N e^{-2 \sigma}}{a} \xi^{0}+\frac{a e^{2 \sigma}}{N} \dot{\xi}^{1}, \\
B & \rightarrow B-\frac{N e^{\sigma}}{a} \xi^{0}+\frac{a e^{-\sigma}}{N} \dot{\xi}, \\
\psi & \rightarrow \psi+2 N(H+2 \Sigma) \xi^{0}+2 \partial_{x}^{2} \xi^{1}, \\
\beta & \rightarrow \beta+e^{3 \sigma} \xi^{1}+e^{-3 \sigma} \xi, \\
\tau & \rightarrow \tau+2 N(H-\Sigma) \xi^{0}, \\
E & \rightarrow E+2 \xi, \\
\pi^{0} & \rightarrow \pi^{0}+\xi^{0}, \\
\pi^{1} & \rightarrow \pi^{1}+\xi^{1}, \\
\pi & \rightarrow \pi+\xi,
\end{aligned}
$$

while for the odd perturbations, we have

$$
\begin{aligned}
v & \rightarrow v+\frac{a e^{-\sigma}}{N} \dot{\xi}_{\text {odd }}, \\
\lambda & \rightarrow \lambda+e^{-3 \sigma} \xi_{\text {odd }}, \\
h & \rightarrow h+2 \xi_{\text {odd }}, \\
\pi_{\text {odd }} & \rightarrow \pi_{\text {odd }}+\xi_{\text {odd }} .
\end{aligned}
$$

Using these transformations, we first define gauge invariant variables which do not refer to the Stückelberg fields. For the even perturbations, we introduce

$$
\begin{aligned}
& \hat{\Phi}=\Phi-\frac{1}{2 N} \partial_{t}\left(\frac{\tau}{H-\Sigma}\right) \\
& \hat{\chi}=\chi+\frac{e^{-2 \sigma}}{2 a(H-\Sigma)} \tau-\frac{a e^{2 \sigma}}{N} \partial_{t}\left[e^{-3 \sigma}\left(\beta-\frac{e^{-3 \sigma}}{2} E\right)\right], \\
& \hat{B}=B+\frac{e^{\sigma}}{2 a(H-\Sigma)} \tau-\frac{a e^{-\sigma}}{2 N} \dot{E}, \\
& \hat{\psi}=\psi-\frac{H+2 \Sigma}{H-\Sigma} \tau-e^{-3 \sigma} \partial_{x}^{2}\left(2 \beta-e^{-3 \sigma} E\right),
\end{aligned}
$$


while for the odd perturbations, we have

$$
\begin{aligned}
& \hat{v}=v-\frac{a e^{-\sigma}}{2 N} \dot{h}, \\
& \hat{\lambda}=\lambda-\frac{e^{-3 \sigma}}{2} h .
\end{aligned}
$$

The variables defined above are not sufficient to account for all of the physical degrees of freedom. Using the Stückelberg perturbations, we can define three even variables

$$
\begin{aligned}
& \hat{\tau}_{\pi}=\pi^{0}-\frac{\tau}{2 N(H-\Sigma)}, \\
& \hat{\beta}_{\pi}=\pi^{1}-e^{-3 \sigma}\left(\beta-\frac{e^{-3 \sigma}}{2} E\right), \\
& \hat{E}_{\pi}=\pi-\frac{1}{2} E
\end{aligned}
$$

and one odd variable

$$
\hat{h}_{\pi}=\pi_{\text {odd }}-\frac{1}{2} h
$$

The gauge invariant variables defined in Eqs.(23) and (24) originate only from the perturbations of the physical metric, thus are already present in the GR case [30]. The additional degrees in Eqs.(25) and (26) are the physical degrees of freedom associated with the additional degrees of the massive graviton and arise from the breaking of general coordinate invariance by the nonzero expectation value of the Stückelberg fields.

\section{B. Odd perturbations}

We start by the action for the odd perturbations only. The perturbed metric we consider is, from (8) and (16),

$$
d s_{\text {odd }}^{2}=-N^{2} d t^{2}+2 a e^{-\sigma} N v_{i} d t d y^{i}+a^{2} e^{4 \sigma} d x^{2}+2 a^{2} e^{\sigma} \partial_{x} \lambda_{i} d x d y^{i}+a^{2} e^{-2 \sigma}\left(\delta_{i j}+\partial_{(i} h_{j)}\right) d y^{i} d y^{j},
$$

while for the Stückelberg fields, we consider

$$
\phi^{0}=t, \quad \phi^{1}=x, \quad \phi^{i}=y^{i}+\pi^{i} .
$$

After using these decompositions in the action (10), then switching to the gauge invariant variables defined in Eqs. (24) and (26), the resulting action depends on the three perturbations $\left(\hat{v}, \hat{\lambda}, \hat{h}_{\pi}\right)$. Among these, $\hat{v}$ does not have any time derivatives and can be removed by solving the constraint equation. In general relativity, this operation also removes $\hat{h}_{\pi}$ and the final action can be written in terms of $\hat{\lambda}$ only. In the nonlinear theory of massive gravity however, we expect that $\hat{h}_{\pi}$ remains in the action as an extra degree of freedom coming from the Stückelberg sector.

We Fourier transform perturbations as

$$
\delta\left(t, x, y^{i}\right)=\int \frac{d k_{L} d k_{T}^{2}}{(2 \pi)^{3 / 2}} e^{i\left(k_{L} x+k_{i} y^{i}\right)} \delta\left(t, k_{L}, k_{i}\right)
$$

where $k_{L}$ and $k_{i}$ are the components of the comoving momentum in the $\hat{x}$ and $\hat{y}^{i}$ directions, respectively. Due to the $2 \mathrm{~d}$ rotational symmetry around the $\hat{x}$ axis, the physical results are expected to depend only on the longitudinal component $k_{L}$ and the magnitude of the transverse components $k_{T} \equiv \sqrt{k_{2}^{2}+k_{3}^{2}}$.

Since the quadratic action does not depend on the derivatives of $\hat{v}$, its equation of motion can be solved by

$$
\hat{v}=\frac{a e^{\sigma}}{N\left[p^{2}\left(e^{\sigma}+r\right)+2 e^{2 \sigma} m_{g}^{2} X J_{\phi}^{(y)}\right]}\left[2 m_{g}^{2} X J_{\phi}^{(y)} \dot{\hat{h}}_{\pi}+e^{-2 \sigma} p_{L}^{2}\left(e^{\sigma}+r\right) \frac{d}{d t}\left(e^{3 \sigma} \hat{\lambda}\right)\right],
$$

where $J_{\phi}^{(y)}$ is defined in Eq. (13), while

$$
p_{L} \equiv \frac{k_{L}}{a e^{2 \sigma}}, \quad p_{T} \equiv \frac{k_{T}}{a e^{-\sigma}}, \quad p^{2} \equiv p_{L}^{2}+p_{T}^{2}
$$


are the physical momenta in longitudinal and transverse directions, and the total physical momentum, respectively.

After a further field redefinition,

$$
\mathcal{V}_{1} \equiv-e^{3 \sigma} \hat{\lambda}, \quad \mathcal{V}_{2} \equiv \frac{2 e^{3 \sigma} p_{L}^{2}}{p^{2}} \hat{\lambda}-2 \hat{h}_{\pi},
$$

the quadratic action takes the following form

$$
I_{\text {odd }}^{(2)}=\frac{M_{p}^{2}}{2} \int N d t d k_{L} d^{2} k_{T} a^{3}\left[K_{11} \frac{\left|\dot{\mathcal{V}}_{1}\right|^{2}}{N^{2}}+K_{22}\left|\frac{\dot{\mathcal{V}}_{2}}{N}-\gamma \mathcal{V}_{1}\right|^{2}-\Omega_{11}^{2}\left|\mathcal{V}_{1}\right|^{2}-\Omega_{22}^{2}\left|\mathcal{V}_{2}\right|^{2}-\Omega_{12}^{2}\left(\mathcal{V}_{1}^{\star} \mathcal{V}_{2}+\mathcal{V}_{2}^{\star} \mathcal{V}_{1}\right)\right]
$$

where

$$
\begin{aligned}
K_{11} & =a^{4} e^{-4 \sigma} \frac{p_{L}^{2} p_{T}^{4}}{2 p^{2}} \\
K_{22} & =\frac{m_{g}^{2} a^{4} e^{-2 \sigma} X J_{\phi}^{(y)} p_{T}^{2} p^{2}}{4\left[2 m_{g}^{2} e^{2 \sigma} X J_{\phi}^{(y)}+\left(e^{\sigma}+r\right) p^{2}\right]}, \\
\gamma & =\frac{12 \Sigma p_{L}^{2} p_{T}^{2}}{p^{4}}, \\
\Omega_{11}^{2} & =\frac{a^{4} e^{-4 \sigma} p_{L}^{2} p_{T}^{4}}{2}\left[1+\frac{m_{g}^{2} e^{\sigma} X}{p^{4}}\left(J_{1} p_{L}^{2}+\frac{2 J_{2} p_{T}^{2}}{1+e^{-3 \sigma}}\right)\right], \\
\Omega_{12}^{2} & =\frac{m_{g}^{2} a^{4} X p_{L}^{2} p_{T}^{4}}{4 p^{2}\left(1+e^{3 \sigma}\right)}\left[\left(1+e^{-3 \sigma}\right) J_{1}-2 J_{2}\right], \\
\Omega_{22}^{2} & =\frac{m_{g}^{2} a^{4} X p_{T}^{2}}{8\left(1+e^{3 \sigma}\right)}\left[2 J_{2} p_{L}^{2}+\left(1+e^{-3 \sigma}\right) J_{1} p_{T}^{2}\right],
\end{aligned}
$$

and

$$
\begin{aligned}
& J_{1} \equiv\left(3+3 \alpha_{3}+\alpha_{4}\right)-\left(1+2 \alpha_{3}+\alpha_{4}\right)\left(r+e^{-2 \sigma}\right) X+\left(\alpha_{3}+\alpha_{4}\right) r e^{-2 \sigma} X^{2}, \\
& J_{2} \equiv\left(3+3 \alpha_{3}+\alpha_{4}\right)-\left(1+2 \alpha_{3}+\alpha_{4}\right)\left(r+e^{\sigma}\right) X+\left(\alpha_{3}+\alpha_{4}\right) r e^{\sigma} X^{2} .
\end{aligned}
$$

\section{Even perturbations}

The perturbed metric for the even sector is, from (8) and (16),

$$
\begin{aligned}
d s_{\text {even }}^{2}= & -N^{2}(1+2 \Phi) d t^{2}+2 a e^{2 \sigma} N \partial_{x} \chi d t d x+2 a e^{-\sigma} N \partial_{i} B d t d y^{i} \\
& +a^{2} e^{4 \sigma}(1+\psi) d x^{2}+2 a^{2} e^{\sigma} \partial_{x} \partial_{i} \beta d x d y^{i}+a^{2} e^{-2 \sigma}\left[\delta_{i j}(1+\tau)+\partial_{i} \partial_{j} E\right] d y^{i} d y^{j},
\end{aligned}
$$

while the even perturbations of Stückelberg fields read

$$
\phi^{0}=t+\pi^{0}, \quad \phi^{1}=x+\partial_{x} \pi^{1}, \quad \phi^{i}=y^{i}+\partial^{i} \pi .
$$

Applying these decompositions to the action (11), we then switch to the gauge invariant variables defined in Eqs. (23) and (25). The resulting action is then manifestly gauge invariant and depends on seven perturbations $(\hat{\Phi}, \hat{B}, \hat{\chi}, \hat{\psi}$, $\left.\hat{\tau}_{\pi}, \hat{\beta}_{\pi}, \hat{E}_{\pi}\right)$. The three degrees arising from the $g_{0 \mu}^{(1)}$ perturbations, namely $\hat{\Phi}, \hat{B}$ and $\hat{\chi}$, are non-dynamical, thus can be integrated out. Furthermore, the kinetic term for the $\hat{\tau}_{\pi}$ is proportional to background equations of motion, so this degree is also non-dynamical. We interpret this as the would-be BD ghost, which is eliminated in this theory by construction. In the massless theory (GR), using the constraint equations also removes the degrees $\hat{\beta}_{\pi}, \hat{E}_{\pi}$, leaving only $\hat{\psi}$ in the action, which becomes one of the gravity wave polarizations in the isotropic limit. However, due to the nonzero mass of the graviton, these two degrees are generically dynamical. Thus, the metric perturbations in vacuum has three physical even perturbations. 
Upon expanding the fields in momentum space, the resulting action is formally

$$
I_{\text {even }}^{(2)}=\frac{M_{p}^{2}}{2} \int N d t d k_{L} d^{2} k_{T} a^{3}\left[\frac{\dot{\mathcal{Y}}^{\dagger}}{N} K \frac{\dot{\mathcal{Y}}}{N}-\mathcal{Y}^{\dagger} \Omega^{2} \mathcal{Y}+\mathcal{Z}^{\dagger} \mathcal{A} \mathcal{Y}+\mathcal{Y}^{\dagger} \mathcal{A}^{T} \mathcal{Z}+\mathcal{Z}^{\dagger} \mathcal{B} \frac{\dot{\mathcal{Y}}}{N}+\frac{\dot{\mathcal{Y}}^{\dagger}}{N} \mathcal{B}^{T} \mathcal{Z}+\mathcal{Z}^{\dagger} \mathcal{C} \mathcal{Z}\right.
$$

with explicit expression for the matrices $K, \Omega^{2}, \mathcal{A}, \mathcal{B}$ and $\mathcal{C}$ given in Appendix $\mathbb{B}$. In the above action, the basis $\mathcal{Y}=\left(\hat{\psi}, \hat{\beta}, \hat{E}_{\pi}\right)^{T}$ corresponds to the three dynamical degrees, while $\mathcal{Z}=\left(\hat{\Phi}, \hat{B}, \hat{\chi}, \hat{\tau}_{\pi}\right)^{T}$ contains the four non-dynamical ones. The equation of motion for the latter can be solved to give

$$
\mathcal{Z}=-\mathcal{C}^{-1}\left(\mathcal{A} \mathcal{Y}+\mathcal{B} \frac{\dot{\mathcal{Y}}}{N}\right)
$$

where we assumed $\operatorname{det} \mathcal{C} \neq 0{ }^{5}$. Using this solution in (38), we obtain

$$
I_{\text {even }}^{(2)}=\frac{M_{p}^{2}}{2} \int N d t d k_{L} d^{2} k_{T} a^{3}\left[\frac{\dot{\mathcal{Y}}^{\dagger}}{N} \bar{K} \frac{\dot{\mathcal{Y}}}{N}+\frac{\dot{\mathcal{Y}}^{\dagger}}{N} \bar{M} \mathcal{Y}+\mathcal{Y}^{\dagger} \bar{M}^{T} \frac{\dot{\mathcal{Y}}}{N}-\mathcal{Y}^{\dagger} \bar{\Omega}^{2} \mathcal{Y}\right]
$$

where

$$
\bar{K}=K-\mathcal{B}^{T} \mathcal{C}^{-1} \mathcal{B}, \quad \bar{M}=-\mathcal{B}^{T} \mathcal{C}^{-1} \mathcal{A}, \quad \bar{\Omega}^{2}=\Omega^{2}+\mathcal{A}^{T} \mathcal{C}^{-1} \mathcal{A}
$$

\section{NONLINEAR INSTABILITY OF FLRW SOLUTION}

In this section, we analyze the action for the perturbations studied in the previous section, by considering small anisotropic departure from a FLRW metric. As discussed in [25], quadratic action in Bianchi-I background with small anisotropy, gives information on the nonlinear action in FLRW background.

Assuming $|\sigma| \ll 1$ and $|\Sigma / H| \ll 1$, we introduce the parameter $\epsilon \ll 1$ and expand the quadratic action with respect to $\epsilon$.

The Stückelberg equation of motion at leading order gives,

$$
3+3 \alpha_{3}+\alpha_{4}-2\left(1+2 \alpha_{3}+\alpha_{4}\right) X+\left(\alpha_{3}+\alpha_{4}\right) X^{2}=\mathcal{O}(\epsilon),
$$

implying

$$
\begin{aligned}
J_{\phi}^{(x)} & =-2 X\left[\left(1+2 \alpha_{3}+\alpha_{4}\right)-\left(\alpha_{3}+\alpha_{4}\right) X\right] \sigma+\mathcal{O}\left(\epsilon^{2}\right), \\
J_{\phi}^{(y)} & =X\left[\left(1+2 \alpha_{3}+\alpha_{4}\right)-\left(\alpha_{3}+\alpha_{4}\right) X\right] \sigma+\mathcal{O}\left(\epsilon^{2}\right), \\
J_{1}=J_{2}+\mathcal{O}(\epsilon) & =(1-r) X\left[\left(1+2 \alpha_{3}+\alpha_{4}\right)-\left(\alpha_{3}+\alpha_{4}\right) X\right]+\mathcal{O}(\epsilon) .
\end{aligned}
$$

\section{A. Odd perturbations}

Expanding the action quadratic in odd modes, the terms (34) at leading order in the small anisotropy expansion are obtained as

$$
\begin{aligned}
K_{11} & =\frac{a^{4} p_{L}^{2} p_{T}^{4}}{2 p^{2}}+\mathcal{O}(\epsilon), \\
K_{22} & =\frac{a^{4} p_{T}^{2} M_{G W}^{2}}{4\left(1-r^{2}\right)} \sigma+\mathcal{O}\left(\epsilon^{2}\right), \\
\gamma & =\mathcal{O}(\epsilon), \\
\Omega_{11}^{2} & =K_{11} \times\left(p^{2}+M_{G W}^{2}\right)+\mathcal{O}(\epsilon), \\
\Omega_{12}^{2} & =\mathcal{O}(\epsilon), \\
\Omega_{22}^{2} & =K_{22} \times\left(c_{s}^{2} p^{2}\right)+\mathcal{O}(\epsilon),
\end{aligned}
$$

\footnotetext{
${ }^{5}$ As we show in Sec VI this is a relevant assumption for the cases we study.
} 
where

$$
M_{G W}^{2}=m_{g}^{2}(1-r) X^{2}\left[\left(1+2 \alpha_{3}+\alpha_{4}\right)-\left(\alpha_{3}+\alpha_{4}\right) X\right], \quad c_{s}^{2}=\frac{1-r^{2}}{2 \sigma} .
$$

Thus, at leading order, we identify the mode $\mathcal{V}_{1}$ with one of the gravity wave $(\mathrm{GW})$ polarizations in FLRW background [15]. The extra degree of freedom $\mathcal{V}_{2}$ is massless and has sound speed $c_{s}$. We also note that the leading order kinetic term for $\mathcal{V}_{2}$ may in general become negative, leading to a ghost degree. For $|\sigma| \ll 1$, in order to avoid the ghost and to make $M_{G W}^{2}$ positive, we require

$$
(1-r) \sigma>0
$$

which also agrees with the condition for avoiding the gradient instability of $\mathcal{V}_{2}$, i.e. $c_{s}^{2}>0$.

\section{B. Even perturbations}

For the even sector, the components of the kinetic matrix defined in (41) at leading order are

$$
\begin{aligned}
\bar{K}_{11} & =\frac{p_{T}^{4}}{8 p^{4}}+\mathcal{O}(\epsilon), \\
\bar{K}_{12} & =-\frac{a^{2} M_{G W}^{2} p_{L}^{2}\left(2 p^{2}+p_{T}^{2}\right)}{2 p^{4}\left(1-r^{2}\right)} \sigma+\mathcal{O}\left(\epsilon^{2}\right), \\
\bar{K}_{13} & =-\frac{a^{2} M_{G W}^{2} p_{T}^{2}\left(p^{2}+p_{L}^{2}\right)}{4 p^{4}\left(1-r^{2}\right)} \sigma+\mathcal{O}\left(\epsilon^{2}\right), \\
\bar{K}_{22} & =-\frac{2 a^{4} M_{G W}^{2} p_{L}^{2}}{1-r^{2}} \sigma+\mathcal{O}\left(\epsilon^{2}\right), \\
\bar{K}_{23} & =\frac{a^{4} M_{G W}^{2} p_{L}^{2} p_{T}^{2}\left(p^{4} r^{2}-3 H^{2} M_{G W}^{2}\right)}{3 H^{2} p^{4}\left(1-r^{2}\right)^{2}} \sigma^{2}+\mathcal{O}\left(\epsilon^{3}\right), \\
\bar{K}_{33} & =\frac{a^{4} M_{G W}^{2} p_{T}^{2}}{1-r^{2}} \sigma+\mathcal{O}\left(\epsilon^{2}\right) .
\end{aligned}
$$

Notice that at order $\epsilon$, the first mode has kinetic mixing with the second and third ones. One can rotate the basis to make the kinetic terms diagonal. The leading order terms of the eigenvalues of the matrix $\bar{K}$ can be found to be:

$$
\begin{aligned}
\kappa_{1} & =\frac{p_{T}^{4}}{8 p^{4}}+\mathcal{O}(\epsilon), \\
\kappa_{2} & =-\frac{2 a^{4} M_{G W}^{2} p_{L}^{2}}{1-r^{2}} \sigma+\mathcal{O}\left(\epsilon^{2}\right), \\
\kappa_{3} & =\frac{a^{4} M_{G W}^{2} p_{T}^{2}}{1-r^{2}} \sigma+\mathcal{O}\left(\epsilon^{2}\right) .
\end{aligned}
$$

The only eigenvalue which is non zero in the FLRW limit is the first one, and corresponds to one of the gravity wave polarizations. The remaining two are thus the extra degrees arising from the graviton mass. However, at leading order, the kinetic terms of these two modes are related through

$$
\kappa_{3}=-\frac{p_{T}^{2}}{2 p_{L}^{2}} \kappa_{2},
$$

which implies that for $|\sigma| \ll 1$, the two extra modes cannot simultaneously have positive kinetic terms; one of them is always a ghost.

The dispersion relation for each mode can be obtained by a series of transformations, as described in detail in Appendix [C] Assuming that the condition (46) is satisfied, the dispersion relations are found to be:

$$
\begin{aligned}
& \omega_{1}^{2}=p^{2}+M_{G W}^{2}+\mathcal{O}(\epsilon), \\
& \omega_{2}^{2}=-\left(\frac{1-r^{2}}{24 \sigma}\right)\left[\sqrt{\left(10 p^{2}+p_{T}^{2}\right)^{2}-8 p_{L}^{2} p_{T}^{2}}-\left(2 p^{2}+3 p_{T}^{2}\right)\right], \\
& \omega_{3}^{2}=\left(\frac{1-r^{2}}{24 \sigma}\right)\left[\sqrt{\left(10 p^{2}+p_{T}^{2}\right)^{2}-8 p_{L}^{2} p_{T}^{2}}+\left(2 p^{2}+3 p_{T}^{2}\right)\right] .
\end{aligned}
$$


The first mode corresponds to one of the GW polarizations in the FLRW limit, while the other two are extra degrees of freedom. Note that in this case, the second degree is a ghost. We also stress that dispersion relation for the extra degrees consists only of momentum terms and thus is gapless.

\section{NONLINEAR STABILITY OF ANISOTROPIC FIXED POINT SOLUTION}

In this section, specializing the field space metric to be pure de Sitter $\left(H_{f}=\right.$ const.), we analyze the action for the perturbations studied in Sec IV by considering small deviation from the anisotropic fixed point found in [23] ${ }^{6}$

$$
H_{0}, \sigma_{0}, X_{0} \text { constants, } \quad r_{0}=e^{-2 \sigma_{0}} .
$$

\section{A. Expanding the background for small anisotropy}

Assuming $\left|\sigma-\sigma_{0}\right| \ll 1$ and $\left|\Sigma / H_{0}\right| \ll 1$, we expand the background quantities

$$
\begin{aligned}
\sigma & =\sigma_{0}+\epsilon \sigma_{1}+\mathcal{O}\left(\epsilon^{2}\right), \\
\Sigma & =\epsilon \Sigma_{1}+\mathcal{O}\left(\epsilon^{2}\right), \\
H & =H_{0}+\epsilon H_{1}+\mathcal{O}\left(\epsilon^{2}\right), \\
X & =X_{0}+\epsilon X_{1}+\mathcal{O}\left(\epsilon^{2}\right), \\
r & =r_{0}+\epsilon r_{1}+\mathcal{O}\left(\epsilon^{2}\right) .
\end{aligned}
$$

Note that $H_{f}$ is not expanded since the fiducial metric is set to be de Sitter. Even if the fiducial metric were of general FLRW type, $H_{f}$ would not be expanded as long as the unitary gauge is employed at the background level.

Let us study the background equations order by order. At order $\mathcal{O}\left(\epsilon^{0}\right)$, upon using the characteristic relation for the anisotropic fixed point, $r_{0}=e^{-2 \sigma_{0}}$, or,

$$
X_{0}=\frac{H_{0}}{H_{f}} e^{2 \sigma_{0}}
$$

there are two more equations at this order;

$$
\begin{array}{r}
H_{0}^{2} e^{3 \sigma_{0}}\left(\alpha_{3}+\alpha_{4}\right)-H_{0} H_{f}\left(1+e^{3 \sigma_{0}}\right)\left(1+2 \alpha_{3}+\alpha_{4}\right)+H_{f}^{2}\left(3+3 \alpha_{3}+\alpha_{4}\right)=0, \\
H_{0}^{2}\left[3+\frac{m_{g}^{2}}{H_{f}^{2}} e^{3 \sigma_{0}}\left(1+2 \alpha_{3}+\alpha_{4}\right)\right]-\frac{H_{0} m_{g}^{2}}{H_{f}}\left(1+e^{3 \sigma_{0}}\right)\left(3+3 \alpha_{3}+\alpha_{4}\right)+m_{g}^{2}\left(6+4 \alpha_{3}+\alpha_{4}\right)+\Lambda=0,
\end{array}
$$

the solutions of which, determine the sets of fixed point values for $H_{0}$ and $\sigma_{0}$. However, noting that $\alpha_{3}$ and $\alpha_{4}$ enter these equations linearly, we can obtain a single set of solutions. Furthermore, introducing two mass scales as

$$
\begin{aligned}
& M^{2} \equiv \frac{H_{0} m_{g}^{2}}{3 H_{f}^{3}}\left[H_{0}^{2} e^{3 \sigma_{0}}\left(1+2 e^{3 \sigma_{0}}\right)\left(\alpha_{3}+\alpha_{4}\right)-2 H_{0} H_{f}\left(1+e^{3 \sigma_{0}}+e^{6 \sigma_{0}}\right)\left(1+2 \alpha_{3}+\alpha_{4}\right)\right. \\
&\left.\quad+H_{f}^{2}\left(2+e^{3 \sigma_{0}}\right)\left(3+3 \alpha_{3}+\alpha_{4}\right)\right], \\
& \tilde{M}^{2} \equiv-\frac{3 H_{0} m_{g}^{2}}{2 H_{f}^{3}}\left[H_{0}^{2} e^{6 \sigma_{0}}\left(\alpha_{3}+\alpha_{4}\right)-2 H_{0} H_{f} e^{3 \sigma_{0}}\left(1+2 \alpha_{3}+\alpha_{4}\right)+H_{f}^{2}\left(3+3 \alpha_{3}+\alpha_{4}\right)\right],
\end{aligned}
$$

which, along with the $\mathcal{O}\left(\epsilon^{\prime}\right)$ order background equations, allow us to express all physical background quantity in terms of $H_{0}, \sigma_{0}, M$ and $\tilde{M}$, in mass units of $H_{f}$. Thus, from here on, we choose the $H_{f}=1$ units.

\footnotetext{
6 The global stability of this fixed point solution, shown in [23], can be interpreted as the validity of the cosmic no-hair conjecture [31] in massive gravity. This point was addressed in the context of bigravity in [32].
} 
Upon using the zero order relations above, the $\mathcal{O}(\epsilon)$ equations yield,

$$
\begin{aligned}
X_{1}= & \frac{H_{0} e^{2 \sigma_{0}}}{\tilde{M}^{4}-27 H_{0}^{2}\left(3 M^{2}-\tilde{M}^{2}\right)}\left\{\left[2 \tilde{M}^{4}+27 H_{0}^{2}\left(3 M^{2}+2 \tilde{M}^{2}\right)\right] \sigma_{1}+18 H_{0} \tilde{M}^{2} \Sigma_{1}\right\}, \\
H_{1}= & -\frac{\tilde{M}^{2}}{\tilde{M}^{4}-27 H_{0}^{2}\left(3 M^{2}-\tilde{M}^{2}\right)}\left(27 H_{0} M^{2} \sigma_{1}+2 \tilde{M}^{2} \Sigma_{1}\right), \\
r_{1} e^{2 \sigma_{0}}= & \frac{243\left(3 M^{2}+2 \tilde{M}^{2}\right)\left(3 M^{2}-\tilde{M}^{2}\right) H_{0}^{4}+9 \tilde{M}^{2} H_{0}^{2}\left(81 M^{4}-66 M^{2} \tilde{M}^{2}+4 \tilde{M}^{4}\right)-\tilde{M}^{6}\left(27 M^{2}-2 \tilde{M}^{2}\right)}{\left[\tilde{M}^{4}-27 H_{0}^{2}\left(3 M^{2}-\tilde{M}^{2}\right)\right]\left[\tilde{M}^{4}+9 H_{0}^{2}\left(3 M^{2}-\tilde{M}^{2}\right)\right]} \\
& -\frac{9 H_{0}\left(9 M^{2}-2 \tilde{M}^{2}\right)}{\tilde{M}^{4}-27 H_{0}^{2}\left(3 M^{2}-\tilde{M}^{2}\right)} \Sigma_{1}, \\
\frac{\dot{\Sigma}_{1}}{N_{0}}= & 3 H_{0}\left(\frac{M^{2} \tilde{M}^{2}\left(9 H_{0}^{2}+\tilde{M}^{2}\right)}{H_{0}\left[\tilde{M}^{4}+9 H_{0}^{2}\left(3 M^{2}-\tilde{M}^{2}\right)\right]} \sigma_{1}-\Sigma_{1}\right) .
\end{aligned}
$$

Using these, we can express all order $\mathcal{O}(\epsilon)$ physical quantity in terms of $\sigma_{1}$ and $\Sigma_{1}$ only.

Finally, we present the expansion of the $J$ functions used extensively throughout the text. We use the above expansion procedure in Eqs.(13) and (35), to obtain

$$
\begin{aligned}
& J_{\phi}^{(x)}=\frac{2 H_{0}^{2} \tilde{M}^{2} e^{3 \sigma_{0}}\left(1-e^{3 \sigma_{0}}\right)^{2}}{2\left(1-H_{0}\right)^{2} \tilde{M}^{2} e^{3 \sigma_{0}}+9 M^{2}\left(1-H_{0} e^{3 \sigma_{0}}\right)^{2}}+\mathcal{O}(\epsilon), \\
& J_{\phi}^{(y)}=\frac{9 H_{0}^{2} \tilde{M}^{2} e^{3 \sigma_{0}}\left(1-e^{3 \sigma_{0}}\right)\left[3 M^{2}\left(9 H_{0}^{2}+\tilde{M}^{2}\right) \sigma_{1}+2 H_{0}\left(9 M^{2}-2 \tilde{M}^{2}\right) \Sigma_{1}\right]}{\left[\tilde{M}^{4}-27 H_{0}^{2}\left(3 M^{2}-\tilde{M}^{2}\right)\right]\left[2\left(1-H_{0}\right)^{2} \tilde{M}^{2} e^{3 \sigma_{0}}+9 M^{2}\left(1-H_{0} e^{3 \sigma_{0}}\right)^{2}\right]}+\mathcal{O}\left(\epsilon^{2}\right) \text {, } \\
& J_{1}=\frac{9 H_{0}^{2} M^{2}\left(1-e^{3 \sigma_{0}}\right)^{2}}{2\left(1-H_{0}\right)^{2} \tilde{M}^{2} e^{3 \sigma_{0}}+9 M^{2}\left(1-H_{0} e^{3 \sigma_{0}}\right)^{2}}+\mathcal{O}(\epsilon), \\
& J_{2}=-\frac{27 H_{0}^{2} M^{2} \tilde{M}^{2} e^{3 \sigma_{0}}\left(1-e^{3 \sigma_{0}}\right)\left(9 H_{0}^{2}+\tilde{M}^{2}\right) \sigma_{1}}{\left[\tilde{M}^{4}+9 H_{0}^{2}\left(3 M^{2}-\tilde{M}^{2}\right)\right]\left[2\left(1-H_{0}\right)^{2} \tilde{M}^{2} e^{3 \sigma_{0}}+9 M^{2}\left(1-H_{0} e^{3 \sigma_{0}}\right)^{2}\right]}+\mathcal{O}\left(\epsilon^{2}\right) .
\end{aligned}
$$

\section{B. Odd perturbations}

We now consider the odd sector action in Eqs. (33)-34) and expand the background quantities around the anisotropic fixed point solution, as prescribed in the previous subsection. The coefficients in the action now read,

$$
\begin{aligned}
K_{11} & =\frac{a_{0}^{4} e^{-4 \sigma_{0}} p_{L}^{2} p_{T}^{4}}{2 p^{2}}+\mathcal{O}(\epsilon) \\
K_{22} & =-\frac{3 \tilde{M}^{2} e^{2 \sigma_{0}} a_{0}^{4} p_{T}^{2}\left[3 M^{2}\left(9 H_{0}^{2}+\tilde{M}^{2}\right) \sigma_{1}+2 H_{0}\left(9 M^{2}-2 \tilde{M}^{2}\right) \Sigma_{1}\right]}{4\left(1-e^{6 \sigma_{0}}\right)\left[\tilde{M}^{4}-27 H_{0}^{2}\left(3 M^{2}-\tilde{M}^{2}\right)\right]}+\mathcal{O}\left(\epsilon^{2}\right) \\
\gamma & =\frac{12 p_{L}^{2} p_{T}^{2} \Sigma_{1}}{p_{T}^{4}}+\mathcal{O}\left(\epsilon^{2}\right) \\
\Omega_{11}^{2} & =K_{11} \times\left(p^{2}-\frac{3 M^{2} p_{L}^{2}}{p^{2}}\right)+\mathcal{O}(\epsilon) \\
\Omega_{12}^{2} & =-\frac{3 M^{2} a_{0}^{4} p_{L}^{2} p_{T}^{4}}{4 e^{4 \sigma_{0}} p^{2}}+\mathcal{O}(\epsilon) \\
\Omega_{22}^{2} & =-\frac{3 M^{2} a_{0}^{4} p_{T}^{4}}{8 e^{4 \sigma_{0}}}+\mathcal{O}(\epsilon) .
\end{aligned}
$$

where the components of the physical momentum are now defined at order $\mathcal{O}\left(\epsilon^{0}\right)$, i.e.

$$
p_{L}=\frac{k_{L}}{a_{0} e^{2 \sigma_{0}}}, \quad p_{T}=\frac{k_{T}}{a_{0} e^{-\sigma_{0}}} .
$$


After the small anisotropy expansion, we see that the first derivative mixing term $\gamma K_{22} \sim \mathcal{O}\left(\epsilon^{2}\right)$ and can be neglected. We introduce a new field basis

$$
\overline{\mathcal{V}}_{1}=\sqrt{K_{11}} \mathcal{V}_{1}, \quad \overline{\mathcal{V}}_{2}=\sqrt{K_{22}} \mathcal{V}_{2}
$$

and bring the kinetic terms to canonical form. Note that we assume that there are no ghost degrees, i.e. $K_{11}, K_{22}>0$. Since the contribution from the time derivatives of the rescaling to the mass term is suppressed in the $\epsilon$ expansion, the odd sector action at leading order becomes

$$
I_{\text {odd }}^{(2)} \simeq \frac{M_{p}^{2}}{2} \int N d t d k_{L} d^{2} k_{T} a^{3}\left[\frac{\left|\dot{\overline{\mathcal{V}}}_{1}\right|^{2}}{N^{2}}+\frac{\left|\dot{\overline{\mathcal{V}}}_{2}\right|^{2}}{N^{2}}-\frac{\Omega_{11}^{2}}{K_{11}}\left|\overline{\mathcal{V}}_{1}\right|-\frac{\Omega_{12}^{2}}{\sqrt{K_{11}} \sqrt{K_{22}}}\left(\overline{\mathcal{V}}_{1}^{\star} \overline{\mathcal{V}}_{2}+\overline{\mathcal{V}}_{2}^{\star} \overline{\mathcal{V}}_{1}\right)-\frac{\Omega_{22}^{2}}{K_{22}}\left|\overline{\mathcal{V}}_{2}\right|\right]
$$

It is then straightforward to find the leading order terms in the dispersion relations,

$$
\begin{aligned}
\omega_{1}^{2} & =p^{2}+\mathcal{O}(\epsilon), \\
\omega_{2}^{2} & =\frac{M^{2} p_{T}^{2}\left(e^{-6 \sigma_{0}}-1\right)\left[\tilde{M}^{4}-27 H_{0}^{2}\left(3 M^{2}-\tilde{M}^{2}\right)\right]}{2 \tilde{M}^{2}\left[3 M^{2}\left(9 H_{0}^{2}+\tilde{M}^{2}\right) \sigma_{1}+2 H_{0}\left(9 M^{2}-2 \tilde{M}^{2}\right) \Sigma_{1}\right]}+\mathcal{O}\left(\epsilon^{0}\right) .
\end{aligned}
$$

We see that the dispersion relations for small deviation from the fixed point, are dominated by momentum terms. Therefore, although the kinetic term of one of the odd modes tends to vanish on the fixed point, we cannot, in general, integrate out this mode from the action since no mass-gap is present.

\section{Even perturbations}

The kinetic matrix $\bar{K}$ defined in (41) is considerably bulky for presentation. In terms of the leading order in small anisotropy expansion around the fixed point, the matrix is formally

$$
\bar{K}=\left(\begin{array}{ccc}
\mathcal{O}\left(\epsilon^{0}\right) & \mathcal{O}\left(\epsilon^{0}\right) & \mathcal{O}(\epsilon) \\
\mathcal{O}\left(\epsilon^{0}\right) & \mathcal{O}\left(\epsilon^{0}\right) & \mathcal{O}(\epsilon) \\
\mathcal{O}(\epsilon) & \mathcal{O}(\epsilon) & \mathcal{O}(\epsilon)
\end{array}\right)
$$

Since $\bar{K}_{11} \bar{K}_{22}-\bar{K}_{12}^{2}=\mathcal{O}\left(\epsilon^{0}\right)$, there is only one mode with a vanishing kinetic term on the fixed point. The two $\mathcal{O}\left(\epsilon^{0}\right)$ eigenvalues involve square-roots (since they are solutions to a quadratic equation) and are not suitable for presentation (nor calculation). However, going to a new basis with a non-orthogonal transformation, we can have (relatively) simpler eigenvalues. We introduce a transformation of the form:

$$
R=\left(\begin{array}{ccc}
1 & 0 & 0 \\
\frac{\bar{K}_{12} \bar{K}_{33}-\bar{K}_{13} \bar{K}_{23}}{K_{23}^{2}-\bar{K}_{22} \bar{K}_{33}} & 1 & 0 \\
\frac{\bar{K}_{13} \bar{K}_{22}-\bar{K}_{12} \bar{K}_{23}}{\bar{K}_{23}^{2}-\bar{K}_{22} \bar{K}_{33}} & -\frac{\bar{K}_{23}}{\bar{K}_{33}} & 1
\end{array}\right)
$$

which we use to define new basis $\mathcal{Y}=R \overline{\mathcal{Y}}$. In this basis, the kinetic matrix becomes:

$$
R^{T} \bar{K} R=\left(\begin{array}{ccc}
\kappa_{1} & 0 & 0 \\
0 & \kappa_{2} & 0 \\
0 & 0 & \kappa_{3}
\end{array}\right)=\left(\begin{array}{ccc}
\bar{K}_{11}+\frac{\bar{K}_{13}^{2} \bar{K}_{22}+\bar{K}_{12}^{2} \bar{K}_{33}-2 \bar{K}_{12} \bar{K}_{13} \bar{K}_{23}}{\bar{K}_{23}^{2}-\bar{K}_{22} \bar{K}_{33}} & 0 & 0 \\
0 & \bar{K}_{22}-\frac{\bar{K}_{23}^{2}}{\bar{K}_{33}} & 0 \\
0 & 0 & \bar{K}_{33}
\end{array}\right)
$$

Notice that the eigenvalues $\kappa_{i}$ are not the same as the eigenvalues of the matrix $\bar{K}$, since the transformation $R$ is not orthogonal. However, $R$ has unit determinant, so the determinant of the kinetic matrix does not change. This is 
enough to study the signature of the eigenvalues. The eigenvalues can then be computed to be:

$$
\begin{aligned}
\kappa_{1} & =\left[\frac{8 p^{4}}{p_{T}^{4}}-\frac{8 \tilde{M}^{4}}{\tilde{M}^{4}+9 H_{0}^{2}\left(3 M^{2}-\tilde{M}^{2}\right)}\right]^{-1}+\mathcal{O}(\epsilon), \\
\kappa_{2} & =\frac{2 a_{0}^{4} e^{8 \sigma_{0}} \tilde{M}^{2} p_{L}^{2}\left[9 H_{0}^{2} p^{4}\left(\tilde{M}^{2}-3 M^{2}\right)+\tilde{M}^{4} p_{L}^{2}\left(-2 p^{2}+p_{L}^{2}\right)\right]}{\tilde{M}^{2} p_{L}^{2}\left(\tilde{M}^{2}-3 p^{2}\right)^{2}-9 H_{0}^{2}\left(\tilde{M}^{2}-3 M^{2}\right)\left[6 p^{4}+\tilde{M}^{2}\left(-4 p^{2}+p_{L}^{2}\right)\right]}+\mathcal{O}(\epsilon), \\
\kappa_{3}= & -\frac{3 \tilde{M}^{2} e^{2 \sigma_{0}} a_{0}^{4} p_{T}^{2}\left[3 M^{2}\left(9 H_{0}^{2}+\tilde{M}^{2}\right) \sigma_{1}+2 H_{0}\left(9 M^{2}-2 \tilde{M}^{2}\right) \Sigma_{1}\right]}{\left(1-e^{6 \sigma_{0}}\right)\left[\tilde{M}^{4}-27 H_{0}^{2}\left(3 M^{2}-\tilde{M}^{2}\right)\right]}+\mathcal{O}\left(\epsilon^{2}\right), .
\end{aligned}
$$

The eigenvalues which are non-zero on the fixed point yield the following no-ghost condition on the parameters

$$
\kappa_{1}>0, \quad \kappa_{2}>0 .
$$

The third eigenvalue, which is of order $\mathcal{O}(\epsilon)$, is actually proportional to the kinetic term of a similar mode in the odd sector, with

$$
\kappa_{3}=4 K_{22}^{\text {odd }}+\mathcal{O}(\epsilon)^{2},
$$

where $K_{22}^{\text {odd }}$ is given in the second line of Eq.(58). Satisfying the third no ghost condition $\kappa_{3}>0$ is rather difficult, since it involves evolving quantities $\sigma_{1}$ and $\Sigma_{1}$.

\section{Evading the ghosts}

Since we are looking for a region in the parameter space where none of the degrees of freedom are ghosts, we impose that the eigenvalues of the kinetic matrix in the even sector are positive, i.e.

$$
\kappa_{1}, \kappa_{2}, \kappa_{3}>0
$$

which ensures that the odd sector is also safe.

For the first two kinetic terms in Eq. (66), it is possible to find regions where a given mode with any momentum is not a ghost. The sufficient (but not necessary) conditions we adopt here are,

$$
\tilde{M}^{2}<0, \quad M^{2}<\frac{\tilde{M}^{2}\left(9 H_{0}^{2}-\tilde{M}^{2}\right)}{27 H_{0}^{2}}<0 .
$$

Thus, $\kappa_{1}$ and $\kappa_{2}$ can be made positive, regardless of the momentum of the modes.

With a parameter set satisfying (70), $\kappa_{3}>0$ gives

$$
\operatorname{Sgn}\left(1-e^{\sigma_{0}}\right)\left[-3\left|M^{2}\right|\left(9 H_{0}^{2}-\left|\tilde{M}^{2}\right|\right) \sigma_{1}+2 H_{0}\left(2\left|\tilde{M}^{2}\right|-9\left|M^{2}\right|\right) \Sigma_{1}\right]>0,
$$

which depends linearly on the deviation of the background from its fixed point, i.e. the two dynamical functions $\sigma_{1}$ and $\Sigma_{1}$. This means that regardless of the value of $M^{2}$ and $\tilde{M}^{2}$, there may always be a region where $\sigma_{1}$ and $\Sigma_{1}$ conspire to give a negative $\kappa_{3}$. On the other hand, close to the attractor, the evolution of these two quantities depend on each other. The equation of motion for $\sigma_{1}$ can be written as [23]

$$
\dot{\Sigma}_{1}+3 H_{0} \Sigma_{1}+M_{\sigma}^{2} \sigma_{1}=0
$$

where

$$
M_{\sigma}^{2} \equiv-\frac{3 M^{2} \tilde{M}^{2}\left(9 H_{0}^{2}+\tilde{M}^{2}\right)}{\tilde{M}^{4}+9 H_{0}^{2}\left(3 M^{2}-\tilde{M}^{2}\right)}
$$

First, the condition that the fixed point is stable, i.e. $M_{\sigma}^{2}>0$, combined with the conditions (700), yields

$$
9 H_{0}^{2}-\left|\tilde{M}^{2}\right|>0 \text {. }
$$


We also wish that $\sigma_{1}$ is over-damped, so that there are no out-of-control changes of signs:

$$
9 H_{0}^{2}>4 M_{\sigma}^{2} .
$$

The solutions to Eq.(172) are easy to obtain,

$$
\sigma=e^{-\frac{3}{2} H_{0} t}\left[C_{1} e^{\sqrt{\frac{9}{4} H_{0}^{2}-M_{\sigma}^{2}} t}+C_{2} e^{-\sqrt{\frac{9}{4} H_{0}^{2}-M_{\sigma}^{2}} t}\right] .
$$

At late times, the general solution approaches to the $C_{2}=0$ solution, where we can write

$$
\left.\Sigma_{1}\right|_{t \rightarrow \infty}=\left.\left(-\frac{3}{2} H_{0}+\sqrt{\frac{9}{4} H_{0}^{2}-M_{\sigma}^{2}}\right) \sigma_{1}\right|_{t \rightarrow \infty} .
$$

So, in this regime, the condition (71) can in principle be satisfied by choosing the appropriate sign for $\sigma_{1}$.

As an example, we choose the set of parameters used in [23]

$$
\alpha_{3}=-\frac{1}{20}, \quad \alpha_{4}=1, \quad \Lambda=0, \quad \mu=20,
$$

implying

$$
e^{\sigma} \simeq 0.51, \quad H_{0} \simeq 15.35
$$

As a result, we find,

$$
M^{2}=-45079, \quad \tilde{M}^{2}=-460.66, \quad \frac{\tilde{M}^{2}\left(9 H_{0}^{2}-\tilde{M}^{2}\right)}{27 H_{0}^{2}}=-186.9, \quad M_{\sigma}^{2}=362.10, \quad \frac{9}{4} H_{0}^{2}-M_{\sigma}^{2}=168.10,
$$

which satisfy the no-ghost conditions for the first two modes (70), fixed point stability condition (74), and overdamping condition (75). For this example, the kinetic term for the third mode, along with the late time attractor assumption (77) becomes

$$
\kappa_{3} \simeq-41.85 a_{0}^{4} p_{T}^{2} \sigma_{1} .
$$

Thus, the parameter set (78) does not lead to any ghost degree, provided that $i$. we are close to the late time attractor; ii. we are in the correct side of the fixed point, i.e. $\sigma_{1}<0$.

\section{DISCUSSION}

Since the introduction of the dRGT theory, there has been several attempts to construct a cosmological solution that is fully consistent with the known expansion history of the universe. In the present paper, we have shown that the self-accelerating solutions introduced in [15, 21] have a ghost instability at nonlinear order. A technical source of this problem is that the kinetic terms of three among the five degrees of freedom are exactly proportional to the equation of motion of the temporal Stückelberg field for the self-accelerating branch. These kinetic terms reappear in the cubic order, and their behavior can be understood by a deformation of the FLRW symmetries. These results were previously reported in 25] and are also compatible with the independent analysis of Ref. 33].

The next step is to seek another class of cosmological solutions on which linear perturbations have non-zero (and positive) kinetic terms. To this end, one needs to ensure that the factorized form of the Stückelberg equation of motion is broken, since kinetic terms of some perturbation degrees of freedom are typically proportional to one of the factors in this equation. Since the FLRW symmetry consists of homogeneity and isotropy, there are two possibilities: breaking either homogeneity or isotropy.

In the present paper we have considered the possibility of breaking the FLRW symmetry by introducing anisotropy. With a relatively large anisotropy, we found a healthy region with non-zero and positive kinetic terms for all five perturbation degrees of freedom. This healthy region is in a neighborhood of (but not exactly on) the de Sitter fixed point solution with an anisotropic hidden sector introduced previously in Ref. [23]. To be more precise, signatures of the resulting kinetic terms depend not only on the direction of deformation of the background from the fixed point, but also on the signature of its time derivative. If the initial condition is such that the evolution is close to the fixed point and that the deformation is in the correct direction, then all five graviton polarizations in the anisotropic background can have positive kinetic terms for a range of parameters. 
Exactly on the fixed point, however, quadratic kinetic terms for two of the five degrees vanish, while all other three degrees have positive kinetic terms in a finite region in the parameter space. Hence, for a small deviation from the fixed point, even though appearance of the ghost degrees can be avoided, two degrees of freedom have very small (cubic order) kinetic terms and no mass gap (see Appendix E). This is an indication that these degrees are strongly coupled and remains to be a source of concern.

Nevertheless, the anisotropic FLRW solution studied here is the first calculable example of a stable cosmology in the dRGT theory of nonlinear massive gravity. One of technical advantages of this solution is that the spatial homogeneity and the $\mathrm{SO}(2)$ invariance of the axisymmetric background allows decoupling between even and odd sectors at the linear order. Moreover, the absence of ghost in a neighborhood of this solution may indicate the existence of region without ghost nor strong coupling somewhere between this fixed point solution and the isotropic FLRW solution. We also note that the perturbations around other Bianchi type universes remain unexplored.

Alternatively, one may decide to break the FLRW symmetries by introducing inhomogeneities. For instance, there are such known solutions [13, 34 39], where the background dynamics is FLRW-like (or reduces to FLRW through cosmological Vainshtein mechanism [13]) while the Stückelberg sector is contaminated by spatial inhomogeneities. In principle, these may also evade the FLRW ghost, although in the lack of symmetry, the stability analysis of perturbations becomes technically challenging (although see [40]).

A different approach to break the factorized form of the time Stückelberg equation is to extend the theory, through introduction of new dynamics, e.g. by imposing a dilatation-like symmetry 41] or considering variations of the parameters with a scalar field [42]. Indeed, the resulting cosmological solutions [43 45] can still be fully homogeneous and isotropic, with non-zero kinetic terms for perturbations. The perturbation analysis of these extensions will be addressed in a separate work [46].

\section{Acknowledgments}

We would like to thank Gianmassimo Tasinato for useful discussions. The work of A.E.G, C.L. and S.M. was supported by the World Premier International Research Center Initiative (WPI Initiative), MEXT, Japan. S.M. also acknowledges the support by Grant-in-Aid for Scientific Research 17740134, 19GS0219, 21111006, 21540278, by Japan-Russia Research Cooperative Program.

\section{Appendix A: Nonexistence of less symmetric fixed point solution of Bianchi-I}

In this Appendix, we show that fixed point solutions of Bianchi-I type always have axisymmetry, as long as the expansion is constrained to be isotropic and the fiducial metric is de Sitter. We introduce the generic Bianchi-I metric as

$$
d s^{2}=-N^{2} d t^{2}+\bar{a}^{2}\left[e^{2\left(\sigma_{1}+\sigma_{2}\right)} d x^{2}+e^{-2 \sigma_{1}} d y^{2}+e^{-2 \sigma_{2}} d z^{2}\right],
$$

which, for $\sigma_{1}=\sigma_{2}, \sigma_{1}=-2 \sigma_{2}$ and $\sigma_{2}=-2 \sigma_{1}$, reduces to the axisymmetric metric used in [23], up to redefinition of coordinates.

For the present discussion, the equations of motion for $\sigma_{1}$ and $\sigma_{2}$ are sufficient:

$$
\begin{array}{r}
\frac{1}{3 N}\left(2 \dot{\Sigma}_{1}+\dot{\Sigma}_{2}\right)+H\left(2 \Sigma_{1}+\Sigma_{2}\right)=\frac{m_{g}^{2}}{3}\left(e^{-\sigma_{1}-\sigma_{2}}-e^{\sigma_{1}}\right) X\left[\left(3+3 \alpha_{3}+\alpha_{4}\right)-\left(1+2 \alpha_{3}+\alpha_{4}\right)\left(e^{\sigma_{2}}+r\right) X\right. \\
\left.+\left(\alpha_{3}+\alpha_{4}\right) e^{\sigma_{2}} r X^{2}\right], \\
\frac{1}{3 N}\left(\dot{\Sigma}_{1}+2 \dot{\Sigma}_{2}\right)+H\left(\Sigma_{1}+2 \Sigma_{2}\right)=\frac{m_{g}^{2}}{3}\left(e^{-\sigma_{1}-\sigma_{2}}-e^{\sigma_{2}}\right) X\left[\left(3+3 \alpha_{3}+\alpha_{4}\right)-\left(1+2 \alpha_{3}+\alpha_{4}\right)\left(e^{\sigma_{1}}+r\right) X\right. \\
\left.+\left(\alpha_{3}+\alpha_{4}\right) e^{\sigma_{1}} r X^{2}\right],
\end{array}
$$

where

$$
r \equiv \frac{n}{N X}, \quad X \equiv \frac{\alpha}{a}, \quad H \equiv \frac{\dot{a}}{a N}, \quad \Sigma_{1} \equiv \frac{\dot{\sigma}_{1}}{N}, \quad \Sigma_{2} \equiv \frac{\dot{\sigma}_{2}}{N} .
$$

We now look for fixed points of the type found in [23], namely, which have isotropy in the expansion $\left(\Sigma_{1}=\Sigma_{2}=0\right)$, but not in the normalization $\left(\sigma_{1} \neq 0, \sigma_{2} \neq 0\right)$. Under these conditions, Eq. (A2) can be written as

$$
\begin{aligned}
& \left(e^{-\sigma_{1}-\sigma_{2}}-e^{\sigma_{1}}\right)\left[\left(3+3 \alpha_{3}+\alpha_{4}\right)-\left(1+2 \alpha_{3}+\alpha_{4}\right)\left(e^{\sigma_{2}}+r\right) X+\left(\alpha_{3}+\alpha_{4}\right) e^{\sigma_{2}} r X^{2}\right]=0, \\
& \left(e^{-\sigma_{1}-\sigma_{2}}-e^{\sigma_{2}}\right)\left[\left(3+3 \alpha_{3}+\alpha_{4}\right)-\left(1+2 \alpha_{3}+\alpha_{4}\right)\left(e^{\sigma_{1}}+r\right) X+\left(\alpha_{3}+\alpha_{4}\right) e^{\sigma_{1}} r X^{2}\right]=0 .
\end{aligned}
$$


Let us discuss the solutions to these equations. For Eq. A4 , one of the solutions is

$$
e^{\sigma_{2}}=e^{-2 \sigma_{1}}
$$

implying axial symmetry around $\hat{z}$ direction. Similarly, Eq. A5 has a solution

$$
e^{\sigma_{1}}=e^{-2 \sigma_{2}}
$$

which implies axial symmetry around $\hat{y}$ direction. Both of these solutions correspond to the solutions already studied in [23]. Removing these, (A4) and (A5) become

$$
\begin{aligned}
& \left(3+3 \alpha_{3}+\alpha_{4}\right)-\left(1+2 \alpha_{3}+\alpha_{4}\right)\left(e^{\sigma_{2}}+r\right) X+\left(\alpha_{3}+\alpha_{4}\right) e^{\sigma_{2}} r X^{2}=0 \\
& \left(3+3 \alpha_{3}+\alpha_{4}\right)-\left(1+2 \alpha_{3}+\alpha_{4}\right)\left(e^{\sigma_{1}}+r\right) X+\left(\alpha_{3}+\alpha_{4}\right) e^{\sigma_{1}} r X^{2}=0
\end{aligned}
$$

for which, the solutions $\sigma_{1}$ and $\sigma_{2}$ are the same, implying axial symmetry around $\hat{x}$ direction. The only alternative solution is $X=\left(2+\alpha_{3}\right) /\left[r\left(1+\alpha_{3}\right)\right]$, which is realized only for $\alpha_{4}=1+\alpha_{3}+\alpha_{3}^{2}$. Moreover, the remaining equations require a further tuning between $\alpha_{3}, m_{g}$ and $\Lambda$, so we drop this solution as well.

Thus, we conclude that all the de Sitter fixed points in Bianchi-I with isotropic expansion are axisymmetric.

\section{Appendix B: Explicit action for even modes around axisymmetric Bianchi-I}

In this Appendix, we present the explicit expression for the action quadratic in even modes. After switching to momentum space, the action has the form (38)

$$
I_{\text {even }}^{(2)}=\frac{M_{p}^{2}}{2} \int N d t d k_{L} d^{2} k_{T} a^{3}\left[\frac{\dot{\mathcal{Y}}^{\dagger}}{N} K \frac{\dot{\mathcal{Y}}}{N}-\mathcal{Y}^{\dagger} \Omega^{2} \mathcal{Y}+\mathcal{Z}^{\dagger} \mathcal{A} \mathcal{Y}+\mathcal{Y}^{\dagger} \mathcal{A}^{T} \mathcal{Z}+\mathcal{Z}^{\dagger} \mathcal{B} \frac{\dot{\mathcal{Y}}}{N}+\frac{\dot{\mathcal{Y}}^{\dagger}}{N} \mathcal{B}^{T} \mathcal{Z}+\mathcal{Z}^{\dagger} \mathcal{C} \mathcal{Z}\right.
$$

where the two field vectors are

$$
\mathcal{Y}=\left(\begin{array}{c}
\hat{\psi} \\
\hat{\beta} \\
\hat{E}_{\pi}
\end{array}\right), \quad \mathcal{Z}=\left(\begin{array}{c}
\hat{\Phi} \\
\hat{B} \\
\hat{\chi} \\
\hat{\tau}_{\pi}
\end{array}\right)
$$

while $K$ and $\Omega^{2}$ are $3 \times 3$ real symmetric matrices, $\mathcal{C}$ is a $4 \times 4$ real symmetric matrix, $\mathcal{A}$ and $\mathcal{B}$ are $4 \times 3$ real matrices. Up to background equations, the components of these matrices are

$$
\begin{aligned}
& K=m_{g}^{2} a^{4} X\left(\begin{array}{ccc}
0 & 0 & 0 \\
0 & \frac{e^{6 \sigma} J_{\phi}^{(x)} p_{L}^{2}}{1+e^{2 \sigma} r} & 0 \\
0 & 0 & \frac{e^{-2 \sigma} J_{\phi}^{(y)} p_{T}^{2}}{e^{\sigma}+r}
\end{array}\right) \\
& \Omega^{2}=\frac{m_{g}^{2} a^{4} e^{2 \sigma} X p_{T}^{2}}{e^{6 \sigma}-1}\left[\left(e^{2 \sigma} r-1\right) J_{\phi}^{(x)}-e^{2 \sigma}\left(r-e^{\sigma}\right) J_{\phi}^{(y)}\right]\left(\begin{array}{ccc}
0 & 0 & -\frac{1+e^{-3 \sigma}}{2 a^{2}} \\
0 & e^{4 \sigma} p_{L}^{2} & -e^{\sigma} p_{L}^{2} \\
-\frac{1+e^{-3 \sigma}}{2 a^{2}} & -e^{\sigma} p_{L}^{2} & e^{-2 \sigma} p_{L}^{2}
\end{array}\right) \\
& \mathcal{A}=\left(\begin{array}{ccc}
\mathcal{A}_{11} & \mathcal{A}_{12} & \mathcal{A}_{13} \\
\mathcal{A}_{21} & 0 & 0 \\
0 & 0 & 0 \\
\mathcal{A}_{41} & \mathcal{A}_{42} & \mathcal{A}_{43}
\end{array}\right), \quad \mathcal{B}=\left(\begin{array}{ccc}
\mathcal{B}_{11} & 0 & 0 \\
\mathcal{B}_{21} & 0 & \mathcal{B}_{23} \\
0 & \mathcal{B}_{32} & 0 \\
\mathcal{B}_{41} & \mathcal{B}_{42} & \mathcal{B}_{43}
\end{array}\right), \quad \mathcal{C}=\left(\begin{array}{cccc}
\mathcal{C}_{11} & \mathcal{C}_{12} & \mathcal{C}_{13} & \mathcal{C}_{14} \\
\mathcal{C}_{12} & \mathcal{C}_{22} & \mathcal{C}_{23} & \mathcal{C}_{24} \\
\mathcal{C}_{13} & \mathcal{C}_{23} & \mathcal{C}_{33} & \mathcal{C}_{34} \\
\mathcal{C}_{14} & \mathcal{C}_{24} & \mathcal{C}_{34} & \mathcal{C}_{44}
\end{array}\right)
\end{aligned}
$$


where

$$
\begin{aligned}
& \mathcal{A}_{11}=\frac{1}{2}\left(p_{T}^{2}+m_{g}^{2} e^{-2 \sigma} X J_{\phi}^{(x)}\right), \\
& \mathcal{A}_{12}=m_{g}^{2} a^{2} e^{2 \sigma} X J_{\phi}^{(x)} p_{L}^{2}, \\
& \mathcal{A}_{13}=m_{g}^{2} a^{2} e^{-\sigma} X J_{\phi}^{(y)} p_{T}^{2}, \\
& \mathcal{A}_{21}=-\frac{3}{2} a e^{-\sigma} \Sigma p_{T}^{2}, \\
& \mathcal{A}_{41}=\frac{3 m_{g}^{2} N r X J_{\phi}^{(x)}}{2\left(1-e^{3 \sigma}\right)}\left(H-H_{f} e^{\sigma} X\right), \\
& \mathcal{A}_{42}=\frac{3 m_{g}^{2} a^{2} e^{4 \sigma} N r X p_{L}^{2} J_{\phi}^{(x)}}{1-e^{3 \sigma}}\left(H-H_{f} e^{\sigma} X\right), \\
& \mathcal{A}_{43}=\frac{m_{g}^{2} a^{2} e^{-\sigma} N r X p_{T}^{2}}{e^{\sigma}-r}\left[J_{\phi}^{(y)}\left(H-\Sigma-H_{f} r X\right)+J_{1}\left(H-\Sigma-H_{f} e^{\sigma} X\right)+J_{2}\left(H+2 \Sigma-H_{f} e^{-2 \sigma} X\right)\right] \\
& \mathcal{B}_{11}=H-\Sigma, \\
& \mathcal{B}_{21}=-\frac{1}{2} a e^{-\sigma} p_{T}^{2}, \\
& \mathcal{B}_{23}=-\frac{m_{g}^{2} a^{3} e^{-\sigma} X J_{\phi}^{(y)} p_{T}^{2}}{e^{\sigma}+r} \\
& \mathcal{B}_{32}=-\frac{m_{g}^{2} a^{3} e^{4 \sigma} X J_{\phi}^{(x)} p_{L}^{2}}{1+e^{2 \sigma} r}, \\
& \mathcal{B}_{41}=\frac{1}{2} m_{g}^{2} \operatorname{NrX} J_{\phi}^{(x)}, \\
& \mathcal{B}_{42}=\frac{m_{g}^{2} a^{2} e^{4 \sigma} N r X J_{\phi}^{(x)} p_{L}^{2}}{1+e^{2 \sigma} r}, \\
& \mathcal{B}_{43}=\frac{m_{g}^{2} a^{2} e^{-\sigma} N r X J_{\phi}^{(y)} p_{T}^{2}}{e^{\sigma}+r},
\end{aligned}
$$




$$
\begin{aligned}
\mathcal{C}_{11}= & -6\left(H^{2}-\Sigma^{2}\right), \\
\mathcal{C}_{12}= & a e^{-\sigma} p_{T}^{2}(2 H+\Sigma), \\
\mathcal{C}_{13}= & 2 a e^{2 \sigma} p_{L}^{2}(H-\Sigma) \\
\mathcal{C}_{14}= & -m_{g}^{2} e^{-2 \sigma} N r X^{2} H_{f}\left(J_{\phi}^{(x)}+2 e^{3 \sigma} J_{\phi}^{(y)}\right), \\
\mathcal{C}_{22}= & \frac{1}{2} a^{2} p_{T}^{2}\left(e^{-2 \sigma} p_{L}^{2}+\frac{2 m_{g}^{2} X J_{\phi}^{(y)}}{e^{\sigma}+r}\right) \\
\mathcal{C}_{23}= & -\frac{1}{2} a^{2} e^{\sigma} p_{L}^{2} p_{T}^{2}, \\
\mathcal{C}_{24}= & \frac{m_{g}^{2} a e^{-\sigma} N r^{2} X J_{\phi}^{(y)} p_{T}^{2}}{e^{\sigma}+r}, \\
\mathcal{C}_{33}= & \frac{1}{2} a^{2} p_{L}^{2}\left(e^{4 \sigma} p_{T}^{2}+\frac{2 m_{g}^{2} X J_{\phi}^{(x)}}{e^{-2 \sigma}+r}\right), \\
\mathcal{C}_{34}= & \frac{m_{g}^{2} a e^{4 \sigma} N r^{2} X J_{\phi}^{(x)} p_{L}^{2}}{1+e^{2 \sigma} r}, \\
\mathcal{C}_{44}= & m_{g}^{2} N^{2} r X\left\{r\left(\frac{J_{\phi}^{(x)} p_{L}^{2}}{e^{-2 \sigma}+r}+\frac{J_{\phi}^{(y)} p_{T}^{2}}{e^{\sigma}+r}\right)-2(H-\Sigma)^{2}\left(\frac{e^{\sigma}}{e^{\sigma}-r} J_{1}+J_{\phi}^{(y)}\right)\right. \\
& \quad-\frac{H-\Sigma}{e^{\sigma}-r}\left[(H+2 \Sigma)\left(2 e^{\sigma} J_{2}+2 e^{-2 \sigma}\left(J_{2}-J_{\phi}^{(x)}\right)-J_{\phi}^{(x)} r\right)-H_{f} e^{-2 \sigma} X\left(2 e^{4 \sigma} J_{1}+2 e^{\sigma} J_{2}-J_{\phi}^{(x)} r\right)\right] \\
& \left.-H_{f} e^{-2 \sigma} X(H+2 \Sigma)\left(J_{\phi}^{(x)}-\frac{2 e^{\sigma}\left(J_{2}-J_{\phi}^{(x)}\right)}{e^{\sigma}-r}\right)-\left(J_{\phi}^{(x)}+2 J_{\phi}^{(y)}\right) \frac{\dot{H}}{N}-2\left(J_{\phi}^{(x)}-J_{\phi}^{(y)}\right) \frac{\dot{\Sigma}}{N}\right\}
\end{aligned}
$$

\section{Appendix C: Diagonalizing the action for even modes near FLRW solutions}

In this Appendix, we analyze the quadratic action for even modes around a Bianchi-I background solution, em-

ploying the small anisotropy expansion. We obtain a simple action for the even modes, through a series of field redefinitions and transformations.

\section{Applying small anisotropy expansion}

We start with the action (40) in the small anisotropy expansion as discussed in Sec VB

$$
I_{\text {even }}^{(2)}=\frac{M_{p}^{2}}{2} \int N d t d k_{L} d^{2} k_{T} a^{3}\left[\frac{\dot{\mathcal{Y}}_{1}^{\dagger}}{N} K_{1} \frac{\dot{\mathcal{Y}}_{1}}{N}+\frac{\dot{\mathcal{Y}}_{1}^{\dagger}}{N} M_{1} \mathcal{Y}_{1}+\mathcal{Y}_{1}^{\dagger} M_{1}^{T} \frac{\dot{\mathcal{Y}}_{1}}{N}-\mathcal{Y}_{1}^{\dagger} \Omega_{1}^{2} \mathcal{Y}_{1}\right]
$$

Since we will be applying several transformations, it is useful to keep track of each step, so we change the notation such that $\mathcal{Y} \rightarrow \mathcal{Y}_{1}, \bar{K} \rightarrow K_{1}, \bar{M} \rightarrow M_{1}$ and $\bar{\Omega}^{2} \rightarrow \Omega_{1}^{2}$. The leading order terms of the matrix components are given 
by

$$
\begin{aligned}
& \left(K_{1}\right)_{11}=\frac{p_{T}^{4}}{8 p^{4}}+\mathcal{O}(\epsilon), \\
& \left(K_{1}\right)_{12}=-\frac{a^{2} M_{G W}^{2} p_{L}^{2}\left(2 p^{2}+p_{T}^{2}\right)}{2 p^{4}\left(1-r^{2}\right)} \sigma+\mathcal{O}\left(\epsilon^{2}\right), \\
& \left(K_{1}\right)_{13}=-\frac{a^{2} M_{G W}^{2} p_{T}^{2}\left(p^{2}+p_{L}^{2}\right)}{4 p^{4}\left(1-r^{2}\right)} \sigma+\mathcal{O}\left(\epsilon^{2}\right), \\
& \left(K_{1}\right)_{22}=-\frac{2 a^{4} M_{G W}^{2} p_{L}^{2}}{1-r^{2}} \sigma+\mathcal{O}\left(\epsilon^{2}\right), \\
& \left(K_{1}\right)_{23}=\frac{a^{4} M_{G W}^{2} p_{L}^{2} p_{T}^{2}\left(p^{4} r^{2}-3 H^{2} M_{G W}^{2}\right)}{3 H^{2} p^{4}\left(1-r^{2}\right)^{2}} \sigma^{2}+\mathcal{O}\left(\epsilon^{3}\right), \\
& \left(K_{1}\right)_{33}=\frac{a^{4} M_{G W}^{2} p_{T}^{2}}{1-r^{2}} \sigma+\mathcal{O}\left(\epsilon^{2}\right) .
\end{aligned}
$$

$$
\begin{aligned}
& \left(M_{1}\right)_{11}=\frac{p_{T}^{4}}{8 H p^{2}}+\mathcal{O}(\epsilon) \\
& \left(M_{1}\right)_{12}=-\frac{a^{2} M_{G W}^{2} p_{L}^{2}}{12 H p^{4}\left(1-r^{2}\right)}\left[6 p^{2} p_{T}^{2}+2 p^{2}\left(2 p^{2}+3 p_{T}^{2}\right) r+3 p_{T}^{4} r^{2}\right] \sigma+\mathcal{O}\left(\epsilon^{2}\right), \\
& \left(M_{1}\right)_{13}=-\frac{a^{2} M_{G W}^{2} p_{T}^{2}}{12 H p^{4}\left(1-r^{2}\right)}\left[-3 p^{2} p_{T}^{2}+p^{2}\left(p^{2}+3 p_{L}^{2}\right) r+3 p_{T}^{4} r^{2}\right] \sigma+\mathcal{O}\left(\epsilon^{2}\right), \\
& \left(M_{1}\right)_{21}=-\frac{a^{2} M_{G W}^{2} p_{L}^{2}}{6 H p^{2}\left(1-r^{2}\right)}\left(2 p^{2} r+3 p_{T}^{2}\right) \sigma+\mathcal{O}\left(\epsilon^{2}\right), \\
& \left(M_{1}\right)_{22}=-\frac{2 a^{4} M_{G W}^{2} p_{L}^{4} r}{3 H\left(1-r^{2}\right)} \sigma+\mathcal{O}\left(\epsilon^{2}\right) \\
& \left(M_{1}\right)_{23}=-\frac{2 a^{4} M_{G W}^{2} p_{L}^{2} p_{T}^{2} r}{3 H\left(1-r^{2}\right)} \sigma+\mathcal{O}\left(\epsilon^{2}\right) \\
& \left(M_{1}\right)_{31}=\frac{a^{2} M_{G W}^{2} p_{T}^{2}}{12 H p^{2}\left(1-r^{2}\right)}\left(2 p^{2} r+3 p_{T}^{2}\right) \sigma+\mathcal{O}\left(\epsilon^{2}\right) \\
& \left(M_{1}\right)_{32}=\frac{a^{4} M_{G W}^{2} p_{L}^{2} p_{T}^{2} r}{3 H\left(1-r^{2}\right)} \sigma+\mathcal{O}\left(\epsilon^{2}\right) \\
& \left(M_{1}\right)_{33}=\frac{a^{4} M_{G W}^{2} p_{T}^{4} r}{3 H\left(1-r^{2}\right)} \sigma+\mathcal{O}\left(\epsilon^{2}\right)
\end{aligned}
$$

$$
\begin{aligned}
& \left(\Omega_{1}^{2}\right)_{11}=\frac{M_{G W}^{2}}{6}+\mathcal{O}(\epsilon), \\
& \left(\Omega_{1}^{2}\right)_{12}=\frac{1}{3} a^{2} M_{G W}^{2} p_{L}^{2}+\mathcal{O}(\epsilon), \\
& \left(\Omega_{1}^{2}\right)_{13}=-\frac{1}{6} a^{2} M_{G W}^{2} p_{T}^{2}+\mathcal{O}(\epsilon), \\
& \left(\Omega_{1}^{2}\right)_{22}=\frac{1}{6} a^{4} M_{G W}^{2} p_{L}^{2}\left(3 p^{2}+p_{L}^{2}\right)+\mathcal{O}(\epsilon), \\
& \left(\Omega_{1}^{2}\right)_{23}=\frac{1}{6} a^{4} M_{G W}^{2} p_{L}^{2} p_{T}^{2}+\mathcal{O}(\epsilon), \\
& \left(\Omega_{1}^{2}\right)_{33}=\frac{1}{6} a^{4} M_{G W}^{2} p_{T}^{2}\left(3 p^{2}+p_{T}^{2}\right)+\mathcal{O}(\epsilon),
\end{aligned}
$$

Although the mixing matrix $M_{1}$ can be made anti-symmetric by adding boundary terms, we keep it for now, since the following transformations will spoil its symmetries anyway. 


\section{Diagonalizing the kinetic matrix}

We start by diagonalizing the kinetic matrix. To achieve this, we apply the following field transformation,

$$
\mathcal{Y}_{2}=R_{1}^{-1} \mathcal{Y}_{1}
$$

where the transformation matrix is

$$
R_{1}=\left(\begin{array}{ccc}
1 & 0 & 0 \\
\frac{\left(K_{1}\right)_{12}\left(K_{1}\right)_{33}-\left(K_{1}\right)_{13}\left(K_{1}\right)_{23}}{\left(K_{1}\right)_{23}^{2}-\left(K_{1}\right)_{22}\left(K_{1}\right)_{33}} & 1 & 0 \\
\frac{\left(K_{1}\right)_{13}\left(K_{1}\right)_{22}-\left(K_{1}\right)_{12}\left(K_{1}\right)_{23}}{\left(K_{1}\right)_{23}^{2}-\left(K_{1}\right)_{22}\left(K_{1}\right)_{33}} & -\frac{\left(K_{1}\right)_{23}}{\left(K_{1}\right)_{33}} & 1
\end{array}\right)
$$

and $\operatorname{det}\left(R_{1}\right)=1$. We stress that $R_{1}$ is not orthogonal. The transformed action takes the form

$$
I_{\text {even }}^{(2)}=\frac{M_{p}^{2}}{2} \int N d t d k_{L} d^{2} k_{T} a^{3}\left[\frac{\dot{\mathcal{Y}}_{2}^{\dagger}}{N} K_{2} \frac{\dot{\mathcal{Y}}_{2}}{N}+\frac{\dot{\mathcal{Y}}_{2}^{\dagger}}{N} M_{2} \mathcal{Y}_{2}+\mathcal{Y}_{2}^{\dagger} M_{2}^{T} \frac{\dot{\mathcal{Y}}_{2}}{N}-\mathcal{Y}_{2}^{\dagger} \Omega_{2}^{2} \mathcal{Y}_{2}\right]
$$

where the new matrices are given by

$$
\begin{aligned}
& K_{2}=R_{1}^{T} K_{1} R_{1} \\
& M_{2}=R_{1}^{T} M_{1} R_{1}+R_{1}^{T} K_{1} \frac{\dot{R}_{1}}{N} \\
& \Omega_{2}^{2}=R_{1}^{T} \Omega_{1}^{2} R_{1}-\frac{\dot{R}_{1}^{T}}{N} M_{1} R_{1}-R_{1}^{T} M_{1}^{T} \frac{\dot{R}_{1}}{N}-\frac{\dot{R}_{1}^{T}}{N} K_{1} \frac{\dot{R}_{1}}{N} .
\end{aligned}
$$

For the vacuum configuration with $\Lambda \neq 0$, the order of time derives can be determined from the background equations in Sec III as

$$
\dot{H}=\mathcal{O}\left(\epsilon^{2}\right), \quad \dot{p}_{L}=-H N p_{L}+\mathcal{O}(\epsilon), \quad \dot{p}_{T}=-H N p_{T}+\mathcal{O}(\epsilon), \quad \dot{M}_{G W}=-M_{G W} \frac{\dot{r}}{2(1-r)}+\mathcal{O}(\epsilon) .
$$

Using these, we can obtain the components of the new matrices as:

$$
\begin{aligned}
\left(K_{2}\right)_{11} & =\frac{p_{T}^{4}}{8 p^{4}}+\mathcal{O}(\epsilon) \\
\left(K_{2}\right)_{22} & =-\frac{2 a^{4} M_{G W}^{2} p_{L}^{2}}{1-r^{2}} \sigma+\mathcal{O}\left(\epsilon^{2}\right) \\
\left(K_{2}\right)_{33} & =\frac{a^{4} M_{G W}^{2} p_{T}^{2}}{1-r^{2}} \sigma+\mathcal{O}\left(\epsilon^{2}\right)
\end{aligned}
$$




$$
\begin{aligned}
&\left(M_{2}\right)_{11}= \frac{p_{T}^{4}}{8 H p^{2}}+\mathcal{O}(\epsilon) \\
&\left(M_{2}\right)_{12}=-\frac{a^{2} M_{G W}^{2} p_{L}^{2} p_{T}^{2}\left(2 p^{2}+p_{T}^{2} r\right)}{4 H p^{4}(1-r)} \sigma+\mathcal{O}\left(\epsilon^{2}\right), \\
&\left(M_{2}\right)_{13}= \frac{a^{2} M_{G W}^{2} p_{T}^{4}\left(p^{2}-p_{T}^{2} r\right)}{4 H p^{4}(1-r)} \sigma+\mathcal{O}\left(\epsilon^{2}\right), \\
&\left(M_{2}\right)_{21}=-\frac{a^{2} M_{G W}^{2} p_{L}^{2} p_{T}^{2}}{2 H p^{2}(1-r)} \sigma+\mathcal{O}\left(\epsilon^{2}\right), \\
&\left(M_{2}\right)_{22}=-\frac{2 a^{4} M_{G W}^{2} p_{L}^{4} r}{3 H\left(1-r^{2}\right)} \sigma+\mathcal{O}\left(\epsilon^{2}\right), \\
&\left(M_{2}\right)_{23}=-\frac{2 a^{4} M_{G W}^{2} p_{L}^{2} p_{T}^{2} r}{3 H\left(1-r^{2}\right)} \sigma+\mathcal{O}\left(\epsilon^{2}\right), \\
&\left(M_{2}\right)_{31}=\frac{a^{2} M_{G W}^{2} p_{T}^{4}}{4 H p^{2}(1-r)} \sigma+\mathcal{O}\left(\epsilon^{2}\right), \\
&\left(M_{2}\right)_{32}=\frac{a^{4} M_{G W}^{2} p_{L}^{2} p_{T}^{2} r}{3 H\left(1-r^{2}\right)} \sigma+\mathcal{O}\left(\epsilon^{2}\right), \\
&\left(M_{2}\right)_{33}=\frac{a^{4} M_{G W}^{2} p_{T}^{4} r}{3 H\left(1-r^{2}\right)} \sigma+\mathcal{O}\left(\epsilon^{2}\right), \\
&\left(\Omega_{2}\right)_{11}=\frac{M_{G W}^{2} p^{4}}{8 p^{4}}+\mathcal{O}(\epsilon), \\
&\left(\Omega_{2}\right)_{12}=\mathcal{O}_{(\epsilon)} \\
&\left(\Omega_{2}\right)_{13}=\mathcal{O}_{(\epsilon)} \\
&\left(\Omega_{2}\right)_{22}=\frac{1}{6} a^{4} M_{G W}^{2} p_{L}^{2}\left(3 p^{2}+p_{L}^{2}\right)+\mathcal{O}(\epsilon), \\
&\left(\Omega_{2}\right)_{23}=\frac{1}{6} a^{4} M_{G W}^{2} p_{L}^{2} p_{T}^{2}+\mathcal{O}(\epsilon), \\
&\left(\Omega_{2}\right)_{33}=\frac{1}{6} a^{4} M_{G W}^{2} p_{T}^{2}\left(3 p^{2}+p_{T}^{2}\right)+\mathcal{O}(\epsilon),
\end{aligned}
$$

\section{Normalizing the kinetic matrix}

We now do the following field rescaling,

$$
\mathcal{Y}_{3}=R_{2}^{-1} \mathcal{Y}_{2},
$$

where

$$
R_{2}=\operatorname{diag}\left[2 \sqrt{2} \frac{p^{2}}{p_{T}^{2}}, \sqrt{\frac{1-r^{2}}{2 \sigma}} \frac{1}{a^{2} M_{G W} p_{L}}, \sqrt{\frac{1-r^{2}}{\sigma}} \frac{1}{a^{2} M_{G W} p_{T}}\right],
$$

where we assumed $(1-r) \sigma>0$. With this transformation, the action becomes

$$
I_{\text {even }}^{(2)}=\frac{M_{p}^{2}}{2} \int N d t d k_{L} d^{2} k_{T} a^{3}\left[\frac{\dot{\mathcal{Y}}_{3}^{\dagger}}{N} K_{3} \frac{\dot{\mathcal{Y}}_{3}}{N}+\frac{\dot{\mathcal{Y}}_{3}^{\dagger}}{N} M_{3} \mathcal{Y}_{3}+\mathcal{Y}_{3}^{\dagger} M_{3}^{T} \frac{\dot{\mathcal{Y}}_{3}}{N}-\mathcal{Y}_{3}^{\dagger} \Omega_{3}^{2} \mathcal{Y}_{3}\right]
$$

where the new matrices are

$$
\begin{aligned}
& K_{3}=R_{2}^{T} K_{2} R_{2}, \\
& M_{3}=R_{2}^{T} M_{2} R_{2}+R_{2}^{T} K_{2} \frac{\dot{R}_{2}}{N}, \\
& \Omega_{3}^{2}=R_{2}^{T} \Omega_{2}^{2} R_{2}-\frac{\dot{R}_{2}^{T}}{N} M_{2} R_{2}-R_{2}^{T} M_{2}^{T} \frac{\dot{R}_{2}}{N}-\frac{\dot{R}_{2}^{T}}{N} K_{2} \frac{\dot{R}_{2}}{N},
\end{aligned}
$$


with components,

$$
\begin{aligned}
& K_{3}=\left(\begin{array}{ccc}
1+\mathcal{O}(\epsilon) & \mathcal{O}\left(\epsilon^{3 / 2}\right) & \mathcal{O}\left(\epsilon^{3 / 2}\right) \\
\mathcal{O}\left(\epsilon^{3 / 2}\right) & -1+\mathcal{O}(\epsilon) & \mathcal{O}\left(\epsilon^{3 / 2}\right) \\
\mathcal{O}\left(\epsilon^{3 / 2}\right) & \mathcal{O}\left(\epsilon^{3 / 2}\right) & 1+\mathcal{O}(\epsilon)
\end{array}\right) \\
& \left(M_{3}\right)_{11}=\frac{p^{2}}{H}+\mathcal{O}(\epsilon), \\
& \left(M_{3}\right)_{12}=\left(M_{3}\right)_{13}=\left(M_{3}\right)_{21}=\left(M_{3}\right)_{31}=\mathcal{O}(\sqrt{\epsilon}) \text {, } \\
& \left(M_{3}\right)_{22}=H-\frac{p_{L}^{2} r}{3 H}+\frac{\Sigma}{2 \sigma}-\frac{\dot{r}}{N(1+r)}+\mathcal{O}(\epsilon) \text {, } \\
& \left(M_{3}\right)_{23}=-\left(M_{3}\right)_{32}=-\frac{\sqrt{2} p_{L} p_{T} r}{3 H}+\mathcal{O}(\epsilon), \\
& \left(M_{3}\right)_{33}=-H+\frac{p_{T}^{2} r}{3 H}-\frac{\Sigma}{2 \sigma}+\frac{\dot{r}}{N(1+r)}+\mathcal{O}(\epsilon), \\
& \left(\Omega_{3}^{2}\right)_{11}=M_{G W}^{2}+\mathcal{O}(\epsilon) \\
& \left(\Omega_{3}^{2}\right)_{12}=\left(\Omega_{3}^{2}\right)_{13}=\mathcal{O}(\sqrt{\epsilon}) \\
& \left(\Omega_{3}^{2}\right)_{22}=\frac{\left(1-r^{2}\right)\left(3 p^{2}+p_{L}^{2}\right)}{12 \sigma}+\mathcal{O}\left(\epsilon^{0}\right), \\
& \left(\Omega_{3}^{2}\right)_{23}=\frac{\left(1-r^{2}\right) p_{L} p_{T}}{6 \sqrt{2} \sigma}+\mathcal{O}\left(\epsilon^{0}\right) \\
& \left(\Omega_{3}^{2}\right)_{33}=\frac{\left(1-r^{2}\right)\left(3 p^{2}+p_{T}^{2}\right)}{6 \sigma}+\mathcal{O}\left(\epsilon^{0}\right) .
\end{aligned}
$$

\section{Anti-symmetrizing the mixing matrix}

We now add the boundary term:

$$
I_{\text {even }}^{(2)} \rightarrow I_{\text {even }}^{(2)}-\frac{M_{p}^{2}}{4} \int N d t d k_{L} d^{2} k_{T} \frac{1}{N} \frac{d}{d t}\left[a^{3} \mathcal{Y}_{3}^{\dagger}\left(M_{3}+M_{3}^{T}\right) \mathcal{Y}_{3}\right],
$$

giving the new action,

$$
I_{\text {even }}^{(2)}=\frac{M_{p}^{2}}{2} \int N d t d k_{L} d^{2} k_{T} a^{3}\left[\frac{\dot{\mathcal{Y}}_{3}^{\dagger}}{N} K_{3} \frac{\dot{\mathcal{Y}}_{3}}{N}+\frac{\dot{\mathcal{Y}}_{3}^{\dagger}}{N} M_{4} \mathcal{Y}_{3}-\mathcal{Y}_{3}^{\dagger} M_{4} \frac{\dot{\mathcal{Y}}_{3}}{N}-\mathcal{Y}_{3}^{\dagger} \Omega_{4}^{2} \mathcal{Y}_{3}\right]
$$

where the mixing matrix is now antisymmetric

$$
M_{4}=\frac{1}{2}\left(M_{3}-M_{3}^{T}\right)=\left(\begin{array}{ccc}
\mathcal{O}(\epsilon) & \mathcal{O}(\sqrt{\epsilon}) & \mathcal{O}(\sqrt{\epsilon}) \\
\mathcal{O}(\sqrt{\epsilon}) & \mathcal{O}(\epsilon) & -\frac{p_{L} p_{T} r}{2 \sqrt{2} H}+\mathcal{O}(\epsilon) \\
\mathcal{O}(\sqrt{\epsilon}) & \frac{p_{L} p_{T} r}{2 \sqrt{2} H}+\mathcal{O}(\epsilon) & \mathcal{O}(\epsilon)
\end{array}\right)
$$

and the frequency matrix is

$$
\Omega_{4}^{2}=\Omega_{3}^{2}+\frac{1}{2 N a^{3}} \frac{d}{d t}\left[a^{3}\left(M_{3}+M_{3}^{T}\right)\right]=\left(\begin{array}{ccc}
p^{2}+M_{G W}^{2}+\mathcal{O}(\epsilon) & \mathcal{O}(\sqrt{\epsilon}) & \mathcal{O}(\sqrt{\epsilon}) \\
\mathcal{O}(\sqrt{\epsilon}) & \frac{\left(1-r^{2}\right)\left(3 p^{2}+p_{L}^{2}\right)}{12 \sigma}+\mathcal{O}\left(\epsilon^{0}\right) & \frac{\left(1-r^{2}\right) p_{L} p_{T}}{6 \sqrt{2} \sigma}+\mathcal{O}\left(\epsilon^{0}\right) \\
\mathcal{O}(\sqrt{\epsilon}) & \frac{\left(1-r^{2}\right) p_{L} p_{T}}{6 \sqrt{2} \sigma}+\mathcal{O}\left(\epsilon^{0}\right) & \frac{\left(1-r^{2}\right)\left(3 p^{2}+p_{T}^{2}\right)}{6 \sigma}+\mathcal{O}\left(\epsilon^{0}\right)
\end{array}\right) .
$$


At this point, we see that the first mode is decoupled at the leading order, and has the same dispersion relation as GW in FLRW,

$$
\omega_{1}^{2}=p^{2}+M_{G W}^{2}+\mathcal{O}(\epsilon) .
$$

From here on, we concentrate on the remaining two degrees only.

\section{Removing the mixing}

The action for the two degrees is now,

$$
I_{\text {even }, 2,3}^{(2)}=\frac{M_{p}^{2}}{2} \int N d t d k_{L} d^{2} k_{T} a^{3}\left[\frac{\dot{\mathcal{Y}}_{5}^{\dagger}}{N} K_{5} \frac{\dot{\mathcal{Y}}_{5}}{N}+\frac{\dot{\mathcal{Y}}_{5}^{\dagger}}{N} M_{5} \mathcal{Y}_{5}-\mathcal{Y}_{5}^{\dagger} M_{5} \frac{\dot{\mathcal{Y}}_{5}}{N}-\mathcal{Y}_{5}^{\dagger} \Omega_{5}^{2} \mathcal{Y}_{5}\right]
$$

where $\mathcal{Y}_{5}=\left[\left(\mathcal{Y}_{3}\right)_{2},\left(\mathcal{Y}_{3}\right)_{3}\right]$ and

$$
\begin{gathered}
K_{5}=\left(\begin{array}{cc}
-1+\mathcal{O}(\epsilon) & \mathcal{O}\left(\epsilon^{3 / 2}\right) \\
\mathcal{O}\left(\epsilon^{3 / 2}\right) & 1+\mathcal{O}(\epsilon)
\end{array}\right), \\
M_{5}=\frac{p_{L} p_{T} r}{2 \sqrt{2} H}\left(\begin{array}{cc}
0 & -1 \\
1 & 0,
\end{array}\right)+\mathcal{O}(\epsilon) \\
\Omega_{5}^{2}=\frac{\left(1-r^{2}\right)}{12 \sigma}\left(\begin{array}{cc}
\left(3 p^{2}+p_{L}^{2}\right) & \sqrt{2} p_{L} p_{T} \\
\sqrt{2} p_{L} p_{T} & 2\left(3 p^{2}+p_{T}^{2}\right)
\end{array}\right)+\mathcal{O}\left(\epsilon^{0}\right) .
\end{gathered}
$$

The next field transformation is

$$
\mathcal{Y}_{6}=R_{5}^{-1} \mathcal{Y}_{5},
$$

where

$$
R_{5}=\left(\begin{array}{cc}
\cosh [\theta(t)] & \sinh [\theta(t)] \\
\sinh [\theta(t)] & \cosh [\theta(t)]
\end{array}\right)
$$

where the transformation function satisfies

$$
\frac{\dot{\theta}}{N}=-\frac{p_{L} p_{T} r}{2 \sqrt{2} H}
$$

In the new basis, the mixing matrix becomes

$$
M_{6}=R_{5}^{T} M_{5} R_{5}+R_{5}^{T} K_{5} \frac{\dot{R}_{5}^{T}}{N}=\mathcal{O}(\sqrt{\epsilon}),
$$

whereas the frequency matrix reads

$$
\Omega_{6}^{2}=R_{5}^{T} \Omega_{5}^{2} R_{5}-\frac{\dot{R}_{5}^{T}}{N} M_{5} R_{5}-R_{5}^{T} M_{5}^{T} \frac{\dot{R}_{5}}{N}-\frac{\dot{R}_{5}^{T}}{N} K_{5} \frac{\dot{R}_{5}}{N}=R_{5}^{T} \Omega_{5}^{2} R_{5}+\mathcal{O}\left(\epsilon^{0}\right),
$$

Finally, the action is, at the relevant order

$$
I_{\text {even }, 2,3}^{(2)}=\frac{M_{p}^{2}}{2} \int N d t d k_{L} d^{2} k_{T} a^{3}\left[\frac{\dot{\mathcal{Y}}_{6}^{\dagger}}{N} K_{5} \frac{\dot{\mathcal{Y}}_{6}}{N}-\mathcal{Y}_{6}^{\dagger}\left(R_{5}^{T} \Omega_{5}^{2} R_{5}\right) \mathcal{Y}_{6}\right] .
$$




\section{6. $\quad$ Eigenfrequencies}

At this point, to diagonalize the frequency matrix, we still need time dependent transformations, which will inevitably reintroduce the mixing. In that sense, it does not seem possible to diagonalize the system at the level of the Lagrangian. This is very similar to the coupled bosons in external background, where the diagonalization is done at the Hamiltonian level [47]. The extended calculation to include ghost degrees is presented in Appendix D. where the eigenvalues of the frequency matrix turn out to be the same as the eigenfrequencies in the diagonalized Hamiltonian.

Thus, the eigenvalues of $\Omega_{6}$ will actually give us the correct dispersion relations. We diagonalize the matrix $\Omega_{6}$ through,

$$
R_{6}^{T} \Omega_{6} R_{6}=R_{6}^{T} R_{5}^{T} \Omega_{5} R_{5} R_{6}=\operatorname{diag}\left(-\omega_{2}^{2}, \omega_{3}^{2}\right)
$$

where

$$
R_{6}=\left(\begin{array}{cc}
\cosh [\varphi(t)] & \sinh [\varphi(t)] \\
\sinh [\varphi(t)] & \cosh [\varphi(t)]
\end{array}\right)
$$

and $\varphi$ satisfies

$$
\cosh [2(\theta+\varphi)]=\frac{10 p^{2}+p_{T}^{2}}{\sqrt{\left(10 p^{2}+p_{T}^{2}\right)^{2}-8 p_{L}^{2} p_{T}^{2}}}, \quad \sinh [2(\theta+\varphi)]=-\frac{2 \sqrt{2} p_{L} p_{T}}{\sqrt{\left(10 p^{2}+p_{T}^{2}\right)^{2}-8 p_{L}^{2} p_{T}^{2}}}
$$

As a result, the eigenfrequencies are found to be ${ }^{7}$

$$
\begin{aligned}
& \omega_{2}^{2}=-\left(\frac{1-r^{2}}{24 \sigma}\right)\left[\sqrt{\left(10 p^{2}+p_{T}^{2}\right)^{2}-8 p_{L}^{2} p_{T}^{2}}-\left(2 p^{2}+3 p_{T}^{2}\right)\right] \\
& \omega_{3}^{2}=\left(\frac{1-r^{2}}{24 \sigma}\right)\left[\sqrt{\left(10 p^{2}+p_{T}^{2}\right)^{2}-8 p_{L}^{2} p_{T}^{2}}+\left(2 p^{2}+3 p_{T}^{2}\right)\right]
\end{aligned}
$$

\section{Appendix D: Dispersion relations of coupled system with ghosts, in external background}

The action of the modes in Section $\mathrm{VB}$ contains a time dependent and non-diagonal frequency matrix, and one of the degrees has a negative kinetic term. At the level of the Lagrangian, it is not possible to recover a diagonal form. In this Appendix, we extend the formalism of [47] to include ghost degrees, show that the Hamiltonian can be diagonalized and obtain dispersion relations of each degree of freedom.

We start by an action of the form

$$
I=\int d^{3} k d t \mathcal{L}_{k}=\frac{1}{2} \int d^{3} k d t\left[\dot{\phi}^{\dagger}(t, \mathbf{k}) \eta \dot{\phi}(t, \mathbf{k})-\phi^{\dagger}(t, \mathbf{k}) \Omega^{2}(t, \mathbf{k}) \phi(t, \mathbf{k})\right],
$$

where $\phi$ is a $N=N_{1}+N_{2}$ dimensional array of fields, with $N_{1}$ ghosts and $N_{2}$ physical fields. $\Omega^{2}$ is a $N \times N$ real and symmetric matrix, which stays invariant under $\mathbf{k} \rightarrow-\mathbf{k}$. The signature of the kinetic matrix is

$$
\eta_{i j}=\delta_{i j} \begin{cases}-1 & , i, j \leq N_{1} \\ +1 & , i, j>N_{1}\end{cases}
$$

\footnotetext{
7 We remind that in this calculation, we assumed that the second mode is a ghost, i.e. $(1-r) \sigma>0$. Assuming that the third mode is a ghost $(1-r) \sigma<0$, one obtains similar expressions for the eigenfrequencies:

$$
\begin{aligned}
& \omega_{2}^{2}=\left(\frac{r^{2}-1}{24 \sigma}\right)\left[\sqrt{\left(10 p^{2}+p_{T}^{2}\right)^{2}-8 p_{L}^{2} p_{T}^{2}}-\left(2 p^{2}+3 p_{T}^{2}\right)\right], \\
& \omega_{3}^{2}=-\left(\frac{r^{2}-1}{24 \sigma}\right)\left[\sqrt{\left(10 p^{2}+p_{T}^{2}\right)^{2}-8 p_{L}^{2} p_{T}^{2}}+\left(2 p^{2}+3 p_{T}^{2}\right)\right] .
\end{aligned}
$$


The conjugate momentum array can be immediately found as

$$
\pi_{i} \equiv \frac{\partial \mathcal{L}_{k}}{\partial \dot{\phi}_{i}^{\dagger}}=\eta_{i j} \dot{\phi}_{j}, \quad \pi_{i}^{\dagger} \equiv \frac{\partial \mathcal{L}_{k}}{\partial \dot{\phi}_{i}}=\dot{\phi}_{j}^{\dagger} \eta_{j i}
$$

giving the Hamiltonian

$$
H=\int d^{3} k \mathcal{H}_{k}=\frac{1}{2} \int d^{3} k\left[\pi^{\dagger}(t, \mathbf{k}) \eta \pi(t, \mathbf{k})+\phi^{\dagger}(t, \mathbf{k}) \Omega^{2}(t, \mathbf{k}) \phi(t, \mathbf{k})\right] .
$$

The Hamiltonian equations of motion are:

$$
\dot{\phi}=\eta \pi, \quad \dot{\pi}=-\Omega^{2} \phi .
$$

We also define the matrix $C=C(t, \mathbf{k})$ which diagonalizes $\Omega^{2}$ through

$$
C^{T} \Omega^{2} C=\eta \omega^{2} \quad(\text { diagonal })
$$

where $C$ is an element of group $S O\left(N_{2}, N_{1}\right)$, i.e.

$$
C^{T} \eta C=\eta
$$

We also note that $C$ is invariant under $\mathbf{k} \rightarrow-\mathbf{k}$.

It turns out to be useful to include the matrix $C$ in the decomposition of the fields $\phi$ and momenta $\pi$ into mode functions in a basis of $N$ dimensional creation/annihilation operator arrays $\hat{a}$ and $\hat{a}^{\dagger}$ as

$$
\begin{aligned}
\phi(t, \mathbf{k}) & =C(t, \mathbf{k})\left[h(t, \mathbf{k}) \hat{a}_{\mathbf{k}}+h^{\star}(t, \mathbf{k}) \hat{a}_{-\mathbf{k}}^{\dagger}\right], \\
\pi(t, \mathbf{k}) & =\left[C^{T}(t, \mathbf{k})\right]^{-1}\left[\tilde{h}(t, \mathbf{k}) \hat{a}_{\mathbf{k}}+\tilde{h}^{\star}(t, \mathbf{k}) \hat{a}_{-\mathbf{k}}^{\dagger}\right],
\end{aligned}
$$

where mode functions $h$ and their conjugates $\tilde{h}$ are $N \times N$ matrices. With this decomposition, we can rewrite Eqs.(D5) as

$$
\dot{h}=\eta \tilde{h}-\Gamma h, \quad \dot{\tilde{h}}=-\eta \omega^{2} h+\Gamma^{T} \tilde{h}
$$

where $\Gamma=C^{-1} \dot{C}$ corresponds to the rate of change of the transformation $C$.

Using the decomposition (D8), the Hamiltonian density becomes

$$
\mathcal{H}_{k}=\frac{1}{2}\left(\hat{a}_{\mathbf{k}}^{\dagger}, \hat{a}_{-\mathbf{k}}\right)\left(\begin{array}{cc}
\tilde{h}^{\dagger} \eta \tilde{h}+h^{\dagger} \eta \omega^{2} h & \tilde{h}^{\dagger} \eta \tilde{h}^{\star}+h^{\dagger} \eta \omega^{2} h^{\star} \\
\tilde{h}^{T} \eta \tilde{h}+h^{T} \eta \omega^{2} h & \tilde{h}^{T} \eta \tilde{h}^{\star}+h^{T} \eta \omega^{2} h^{\star}
\end{array}\right)\left(\begin{array}{l}
\hat{a}_{\mathbf{k}} \\
\hat{a}_{-\mathbf{k}}^{\dagger}
\end{array}\right) .
$$

Finally, we do a further redefinition, based on the solutions to the equations of motion in adiabatic regime,

$$
h=\frac{1}{\sqrt{2 \omega}}(\alpha+\beta), \quad \tilde{h}=-i \sqrt{\frac{\omega}{2}} \eta(\alpha-\beta),
$$

where $\alpha$ and $\beta$ are $N \times N$ complex matrices and are generalizations of Bogolyubov coefficients. We stress that this redefinition keeps generality.

Using the definition (D11) in the Hamiltonian density (D10), we obtain the Hamiltonian density

$$
\mathcal{H}_{k}=\frac{1}{2}\left(\hat{b}_{\mathbf{k}}^{\dagger}, \hat{b}_{-\mathbf{k}}\right)\left(\begin{array}{cc}
\eta \omega & 0 \\
0 & \eta \omega
\end{array}\right)\left(\begin{array}{l}
\hat{b}_{\mathbf{k}} \\
\hat{b}_{-\mathbf{k}}^{\dagger}
\end{array}\right)
$$

where the new creation/annihilation operators are defined as

$$
\left(\begin{array}{l}
\hat{b}_{\mathbf{k}} \\
\hat{b}_{-\mathbf{k}}^{\dagger}
\end{array}\right) \equiv\left(\begin{array}{cc}
\alpha & \beta^{\star} \\
\beta & \alpha^{\star}
\end{array}\right)\left(\begin{array}{l}
\hat{a}_{\mathbf{k}} \\
\hat{a}_{-\mathbf{k}}^{\dagger}
\end{array}\right)
$$


Since $\eta \omega$ is already diagonal, the Hamiltonian in the new basis is also diagonal. After normal ordering, we end up with

$$
\begin{aligned}
\mathcal{H}_{k} & =\frac{1}{2} \sum_{i=1}^{N}(\eta \omega)_{i} \hat{b}_{i}^{\dagger} \hat{b}_{i} . \\
& =-\frac{1}{2} \sum_{i=1}^{N_{1}} \omega_{i} \hat{b}_{i}^{\dagger} \hat{b}_{i}+\frac{1}{2} \sum_{i=1}^{N_{2}} \omega_{i} \hat{b}_{i}^{\dagger} \hat{b}_{i}
\end{aligned}
$$

This calculation shows that the eigenvalues of the frequency matrix in Eq.(D1) actually correspond to the eigenfrequencies of the modes in the diagonal basis.

\section{Appendix E: The situation of perturbations on the fixed point}

Since the even mode action in Sec VIC is very bulky, it was not possible to obtain the dispersion relations for each degree of freedom, at least not for arbitrary momentum. Observing that the matrix $\bar{M}$ which mixes first derivatives with the fields is of order $\mathcal{O}\left(\epsilon^{0}\right)$, it is then a fair question to ask whether the kinetic term is modified when the action is brought to a diagonal form.

However, this reasoning requires integrating out the field $\mathcal{Y}_{3}=\hat{E}_{\pi}$, a procedure which cannot be performed in our case as the field $\mathcal{Y}_{3}$ does not have any mass-gap. Let us consider this issue in more detail. The term $\mu^{2}$ in the Lagrangian, where $\mathcal{L} \ni-\mu^{2} \mathcal{Y}_{3}^{2}$, is surely of order $\mathcal{O}\left(\epsilon^{0}\right)$ but multiplied by a factor $p_{T}^{4}$. Instead, the kinetic term of this same field $\mathcal{Y}_{3}$ - which tends to exactly vanish on the fixed point - is multiplied only by a factor $p_{T}^{2}$ as shown in Eq. (66). Rescaling the field by $\mathcal{Y}_{3} \rightarrow \tilde{\mathcal{Y}}_{3} / p_{T}$, makes the $\mathcal{O}(\epsilon)$-kinetic term momentum-independent; however, its mass - being proportional to $p_{T}^{2}-$ still tends to vanish for small $p_{T}$. This is tantamount to saying that we cannot in fact describe the mass of the field in the small transverse-momentum limit, that is $p_{T} \rightarrow 0$, because we still have that $\lim _{p_{T} \rightarrow 0}\left(\mu^{2} / p_{T}^{2}\right) \rightarrow 0$ and, consequently, we cannot integrate the mode $\mathcal{Y}_{3}$ any more in this limit.

Therefore, we are led to deduce that we cannot, in general, study the perturbations on the exact-fixed-point solution, as this would lead to the inconsistency of being able to integrate out a mode which actually cannot be integrated out - at least for small $p_{T}$. On the other hand, it makes sense to study that background which is not the fixed point, but close enough to it. We have followed this last procedure in our analysis in this paper.

This situation also implies that, in order to check whether there is a strong-coupling limit on the fixed point solution, we need to analyze the Lagrangian at higher orders (cubic, at least) in the perturbations, and check whether there is indeed any non-trivial strong contribution/back-reaction coming from these higher order terms.

\section{Appendix F: Even sector dispersion relations in the IR}

Unfortunately, for the perturbation analysis around the anisotropic fixed point discussed in Sec.VI the problem is technically very involved, and calculation of the full dispersion relations is very difficult. However, it is possible to finalize the diagonalization in the IR regime. This approach allows us to determine whether the even mode with vanishing kinetic term on the fixed point suffers from strong coupling or not. Notice that we already have one mode (in the odd sector) which has vanishing kinetic term and has no mass gap.

Due to the direction dependence of the background, in the IR regime where the momentum dependence is removed, the dispersion relations still depend on the orientation of the momentum vector. We decompose the momenta as

$$
p_{L}=p \xi, \quad p_{T}=p \sqrt{1-\xi^{2}},
$$

and take the limit $p \rightarrow 0$. Proceeding the same way as we did for the FLRW solution (see Appendix C for details), the diagonalization in this limit reveals,

$$
\omega_{1}=\mathcal{O}\left(p^{0}\right), \quad \omega_{2}=\mathcal{O}\left(p^{0}\right),\left.\quad \omega_{3}\right|_{p \rightarrow 0}=0
$$

where modes labeled 1 and 2 correspond to degrees which have non vanishing kinetic terms on the anisotropic fixed point, while for mode 3 , the kinetic term is zero.

Combining our result from the $2 \mathrm{~d}$ vector sector, we observe that:

- Even though by appropriate choice of parameters, we may avoid ghost degrees, for the two modes which have vanishing kinetic terms, there is no mass gap. Therefore, we expect that they are infinitely strongly coupled. 
- The mode with $\mathcal{O}(1)$ kinetic term in the odd sector is massless on the fixed point, with sound speed $c_{s}=1$.

- The two modes with $\mathcal{O}(1)$ kinetic term in the even sector have masses, but these depend on the orientation of the momentum vector. The gradient part of the dispersion relation was not calculated.

For the two massive modes in the even sector, the mass terms are still to bulky for presentation. We end this section by looking at two extreme examples for the momentum direction.

\section{1. $\xi \rightarrow 1$, momentum is in the $\hat{x}$ direction}

In this case, the momentum is aligned with the privileged direction $\hat{x}$. The masses for this situation is

$$
m_{1}^{2} \simeq-3 M^{2}, \quad m_{2}^{2} \simeq \frac{3 M^{2} \tilde{M}^{2}\left(9 H_{0}^{2}+\tilde{M}^{2}\right)}{9 H_{0}^{2}\left(\tilde{M}^{2}-3 M^{2}\right)-\tilde{M}^{4}} .
$$

If the no-ghost condition (70) and fixed point stability condition (74) are satisfied, both squared-masses are positive.

\section{2. $\xi \rightarrow 0$, momentum is perpendicular to the $\hat{x}$ direction}

In this case, the momentum is along the $y-z$ plane. The masses of the modes then become

$$
\begin{aligned}
& m_{1}^{2} \simeq-\frac{M^{2} \tilde{M}^{2}\left[\tilde{M}^{8}+36 H_{0}^{2} \tilde{M}^{4}\left(\tilde{M}^{2}-3 M^{2}\right)+243 H_{0}^{4}\left(\tilde{M}^{2}-3 M^{2}\right)^{2}\right]}{M^{2} \tilde{M}^{8}+27 H_{0}^{4}\left(\tilde{M}^{2}-3 M^{2}\right)^{2}\left(3 M^{2}+2 \tilde{M}^{2}\right)+18 H_{0}^{2} \tilde{M}^{4}\left(3 M^{4}-4 M^{2} \tilde{M}^{2}+\tilde{M}^{4}\right)} \xi^{2} \\
& m_{2}^{2} \simeq \frac{M^{4}\left[9 H_{0}^{2}\left(\tilde{M}^{2}-3 M^{2}\right)-\tilde{M}^{4}\right]}{4 H_{0}^{2}\left(\tilde{M}^{2}-3 M^{2}\right)^{2}}+\frac{54 H_{0}^{2} M^{2} \tilde{M}^{2}}{9 H_{0}^{2}\left(\tilde{M}^{2}-3 M^{2}\right)-\tilde{M}^{4}}\left[1-\frac{9 H_{0}^{2}\left(\tilde{M}^{2}-3 M^{2}\right)-\tilde{M}^{4}}{12 H_{0}^{2}\left(\tilde{M}^{2}-3 M^{2}\right)}\right] .
\end{aligned}
$$

Again, once the conditions (70) and (74) are imposed, both squared-masses are positive.

[1] M. Fierz and W. Pauli, Proc. Roy. Soc. Lond. A 173, 211 (1939).

[2] H. van Dam, M. J. G. Veltman, Nucl. Phys. B22, 397-411 (1970).

[3] V. I. Zakharov, JETP Lett. 12, 312 (1970).

[4] A. I. Vainshtein, Phys. Lett. B 39, 393 (1972).

[5] D. G. Boulware and S. Deser, Phys. Rev. D 6, 3368 (1972).

[6] C. de Rham, G. Gabadadze and A. J. Tolley, Phys. Rev. Lett. 106, 231101 (2011) arXiv:1011.1232 [hep-th]].

[7] C. de Rham, G. Gabadadze, Phys. Rev. D82, 044020 (2010). arXiv:1007.0443 [hep-th]].

[8] S. F. Hassan and R. A. Rosen, Phys. Rev. Lett. 108, 041101 (2012) arXiv:1106.3344 [hep-th]].

[9] A. Gruzinov, arXiv:1106.3972 [hep-th].

[10] C. de Rham, G. Gabadadze and A. J. Tolley, arXiv:1107.0710 [hep-th].

[11] S. Deser and A. Waldron, arXiv:1212.5835 [hep-th].

[12] A. Higuchi, Nucl. Phys. B 282, 397 (1987).

[13] G. D'Amico, C. de Rham, S. Dubovsky, G. Gabadadze, D. Pirtskhalava and A. J. Tolley, Phys. Rev. D 84, 124046 (2011) arXiv:1108.5231 [hep-th]].

[14] S. F. Hassan, R. A. Rosen and A. Schmidt-May, JHEP 1202, 026 (2012) arXiv:1109.3230 [hep-th]].

[15] A. E. Gumrukcuoglu, C. Lin and S. Mukohyama, JCAP 1203, 006 (2012) arXiv:1111.4107 [hep-th]].

[16] M. Fasiello and A. J. Tolley, JCAP 1211, 035 (2012) arXiv:1206.3852 [hep-th]].

[17] D. Langlois and A. Naruko, Class. Quant. Grav. 29, 202001 (2012) [arXiv:1206.6810 [hep-th]].

[18] C. de Rham and S. Renaux-Petel, JCAP 1301, 035 (2013) arXiv:1206.3482 [hep-th]].

[19] S. Deser, M. Sandora and A. Waldron, arXiv:1301.5621 [hep-th].

[20] C. de Rham, K. Hinterbichler, R. A. Rosen and A. J. Tolley, arXiv:1302.0025 [hep-th].

[21] A. E. Gumrukcuoglu, C. Lin and S. Mukohyama, JCAP 1111, 030 (2011) arXiv:1109.3845 [hep-th]].

[22] A. E. Gumrukcuoglu, S. Kuroyanagi, C. Lin, S. Mukohyama and N. Tanahashi, Class. Quant. Grav. 29, 235026 (2012) arXiv:1208.5975 [hep-th]].

[23] A. E. Gumrukcuoglu, C. Lin and S. Mukohyama, Phys. Lett. B 717, 295 (2012) arXiv:1206.2723 [hep-th]]. 
[24] K. Koyama, G. Niz and G. Tasinato, JHEP 1112, 065 (2011) arXiv:1110.2618 [hep-th]]; G. Tasinato, K. Koyama and G. Niz, arXiv:1210.3627 [hep-th].

[25] A. De Felice, A. E. Gumrukcuoglu and S. Mukohyama, Phys. Rev. Lett. 109, 171101 (2012) [arXiv:1206.2080 [hep-th]].

[26] N. Arkani-Hamed, H. -C. Cheng, M. A. Luty and S. Mukohyama, JHEP 0405, 074 (2004) hep-th/0312099].

[27] N. Arkani-Hamed, H. -C. Cheng, M. A. Luty, S. Mukohyama and T. Wiseman, JHEP 0701, 036 (2007) hep-ph/0507120.

[28] K. -i. Maeda and M. S. Volkov, arXiv:1302.6198 [hep-th].

[29] A. E. Gumrukcuoglu, C. R. Contaldi and M. Peloso, JCAP 0711, 005 (2007) arXiv:0707.4179 [astro-ph]].

[30] B. Himmetoglu, C. R. Contaldi and M. Peloso, Phys. Rev. D 79, 063517 (2009) arXiv:0812.1231 [astro-ph]].

[31] R. M. Wald, Phys. Rev. D 28, 2118 (1983).

[32] Y. Sakakihara, J. Soda and T. Takahashi, arXiv:1211.5976 [hep-th].

[33] G. D'Amico, Phys. Rev. D 86, 124019 (2012) arXiv:1206.3617 [hep-th]].

[34] K. Koyama, G. Niz, G. Tasinato and , Phys. Rev. Lett. 107, 131101 (2011) [arXiv:1103.4708 [hep-th]].

[35] K. Koyama, G. Niz, G. Tasinato and, Phys. Rev. D 84, 064033 (2011) arXiv:1104.2143 [hep-th]].

[36] P. Gratia, W. Hu and M. Wyman, Phys. Rev. D 86, 061504 (2012) arXiv:1205.4241 [hep-th]].

[37] T. Kobayashi, M. Siino, M. Yamaguchi and D. Yoshida, Phys. Rev. D 86, 061505 (2012) arXiv:1205.4938 [hep-th]].

[38] H. Motohashi and T. Suyama, Phys. Rev. D 86, 081502 (2012) arXiv:1208.3019 [hep-th]].

[39] M. S. Volkov, Phys. Rev. D 86, 061502 (2012) arXiv:1205.5713 [hep-th]]; M. S. Volkov, Phys. Rev. D 86, 104022 (2012) arXiv:1207.3723 [hep-th]].

[40] M. Wyman, W. Hu and P. Gratia, arXiv:1211.4576 [hep-th].

[41] G. D'Amico, G. Gabadadze, L. Hui and D. Pirtskhalava, arXiv:1206.4253 [hep-th].

[42] Q. -G. Huang, Y. -S. Piao and S. -Y. Zhou, Phys. Rev. D 86, 124014 (2012) arXiv:1206.5678 [hep-th]].

[43] D. -J. Wu, Y. -S. Piao and Y. -F. Cai, arXiv:1301.4326 [hep-th].

[44] K. Hinterbichler, J. Stokes and M. Trodden, arXiv:1301.4993 [astro-ph.CO].

[45] G. Leon, J. Saavedra and E. N. Saridakis, arXiv:1301.7419] [astro-ph.CO].

[46] A. E. Gumrukcuoglu, K. Hinterbichler, C. Lin, S. Mukohyama and M. Trodden, arXiv:1304.0449 [hep-th].

[47] H. P. Nilles, M. Peloso and L. Sorbo, JHEP 0104, 004 (2001) hep-th/0103202. 